\title{
A novel Calmodulin-interacting Domain of Unknown Function 506 protein represses root hair elongation in Arabidopsis
}

\author{
Sheng Ying ${ }^{1}$ and Wolf Scheible ${ }^{1}$ \\ ${ }^{1}$ Noble Research Institute LLC
}

September 24, 2021

\begin{abstract}
DUF506 proteins are omnipresent in higher plants. Phosphorus (P) stress-inducible AtRXR1/REPRESSOR OF EXCESSIVE ROOT HAIR ELONGATION 1 gene, as the first functionally characterized DUF506 gene, is proved to inhibit root hair elongation through interaction of RXR2/RabD2c GTPase. However, the knowledge of other P-responsive DUF506 genes is still limited. Here, we identify four additional P-inducible DUF506 genes and select two of candidates for functional investigation. Expression analysis results reveal that both of candidates are induced by auxin. At3g07350, the duplicated pair of RXR1, expresses ubiquitously in seedlings under P-stress, whereas At1g62420 is mainly induced in roots. Overexpression and knockout mutants of At1g62420, which is called RXR3, exhibit shorter or longer root hair length, respectively. Cellular imaging results demonstrate RXR3 localizes in root epidermal cells. ChIP, synthetic peptide treatment and qRT-PCR assay results indicate RXR3 is transcriptionally activated by RSL4 or RALF1. BiFC and CaM-binding assay suggest that RXR3 interacts with various $\mathrm{CaMs}$ in presence of $\mathrm{Ca} 2+$. Moreover, the frequencies of $[\mathrm{Ca} 2+]$ cyt in rxr3 mutants are approximately $20 \%$ higher compared to that of wild type. Taken together, our results illustrate a divergent mechanism by which RSL4-directed RXR3 interacts with calmodulin and functions in parallel of RXR1, to prevent root hair excessive growth.
\end{abstract}

\section{INTRODUCTION}

Phosphorus (P) is essential for all life on earth as an indispensable structural component of nucleic acids, membrane phospholipids and energy metabolites. Plants assimilate $\mathrm{P}$ as inorganic phosphate (Pi) from the soil. However, the concentration of Pi in many unfertilized soils is low and limiting for plant growth (Hammond, Broadley, \& White, 2004; Raghothama, 1999). In order to cope with a low P environment, plants have evolved various strategies to improve the acquisition, remobilization and efficient use of $\mathrm{Pi}$, such as altering root morphology, secreting phosphatases and nucleases to mobilize or release Pi from soil insoluble organic sources (Lynch, 2011; Plaxton \& Tran, 2011; Svistoonoff et al., 2007; Williamson, Ribrioux, Fitter, \& Leyser, 2001). One morphological hallmark response of roots to low $\mathrm{P}$ is the increase of density and length of root hairs (RHs), which is thought to maximize the root surface area $(\sim 70 \%)$ and facilitate the absorbance of Pi present in the soil (could be responsible for up to 90\%)(Bates \& Lynch, 2001; Bucher, 2007; Crombez, Motte, \& Beeckman, 2019; Lynch, 2019; Ma, Baskin, Brown, \& Lynch, 2003). Additionally, RHs aid water uptake(Kwasniewski et al., 2016), improve root penetration of hard/drying soil (Choi \& Cho, 2019; Haling et al., 2013), enhance plant tolerance to drought stress (Marin et al., 2020; Xiaomin Zhang et al., 2020), and enable root-microbe interactions (Brown, George, Dupuy, \& White, 2013).

In Arabidopsis, RHs developed from specialized root epidermal cells, called trichoblasts, located outside the intercellular space between two underlying cortical cells (Grierson, Nielsen, Ketelaarc, \& Schiefelbein, 2014). The genetics and molecular mechanisms of root hairs development, i.e. initiation, differentiation, and elongation, have been extensively studied (Bruex et al., 2012; Cho \& Cosgrove, 2002; Hwang, Choi, Cho, \& Cho, 2017; Lin et al., 2015; Parker, Cavell, Dolan, Roberts, \& Grierson, 2000; Salazar-Henao, Vélez-Bermúdez, \& Schmidt, 2016; Schiefelbein, 2000), and have identified a number of key genes. ROOT HAIR DEFECTIVE6 
(RHD6), a basic helix-loop-helix (bHLH) transcription factor (TF), plays central roles in RH development (Masucci \& Schiefelbein, 1994). As downstream bHLH TFs of RHD6, RHD6-LIKE2/4 (RSL2/4), dominantly regulate expression of various RH morphogenic genes (such as EXPANSIN A7/EXPA7, PEROXIDASE7/PER7, and CALCIUM-DEPENDENT PROTEIN KINASE11/CDPK11), through direct binding to root hair-specific cis -elements (RHEs) in their proximal promoter regions, and consequently control the elongation of RHs (Bhosale et al., 2018; Datta, Prescott, \& Dolan, 2015; Hwang et al., 2017; Kim \& Dolan, 2016; Kim, Han, \& Dolan, 2017; Vijayakumar, Datta, \& Dolan, 2016; Yi, Menand, Bell, \& Dolan, 2010).

To date, many phytohormones (e.g. auxin/IAA, ethylene/ACC, Abscisic acid/ABA, Strigolactones/SLs and Jasmonate/JA), as well as chemical molecules (e.g. reactive oxygen species/ROS, $\mathrm{Ca}^{2+}$, and short peptide/RAPID ALKALINIZATION FACTOR1/AtRALF1), have been demonstrated to regulate RH development (Abarca, Franck, \& Zipfel, 2021; Y. Feng et al., 2017; Foreman et al., 2003; Han, Zhang, Yang, \& Hu, 2020; Kapulnik et al., 2011; S. Mangano et al., 2017; Silvina Mangano, Denita-Juarez, Marzol, Borassi, \& Estevez, 2018; Pitts, Cernac, \& Estelle, 1998; Tian, Wang, Gao, Li, \& Luan, 2020; Vissenberg, Claeijs, Balcerowicz, \& Schoenaers, 2020; Wang et al., 2017; Zhu, Martínez Pacheco, Estevez, \& Yu, 2020). Recently, two models have been proposed to illustrate the mechanism of RH elongation when exposed to low external P (Bhosale et al., 2018; Song et al., 2016). For instance, under P-deficiency condition, endogenous auxin level is significantly elevated in the root apex, which leads to activation of AUX1-mediated auxin transport and following the induction of ARF19, eventually stimulate the RSL2 and RSL4 to enhance RH elongation. In RH cell, NADPH oxidase (e.g. RHD2) trigger the accumulation of ROS, which subsequently stimulate the cytosolic $\mathrm{Ca}^{2+}\left(\left[\mathrm{Ca}^{2+}\right]_{\mathrm{cyt}}\right)$ oscillation and gradient, through the activation of cyclic nucleotide-gated channels (CNGCs)(Foreman et al., 2003; Tian et al., 2020).

Domains of Unknown Function 506 (DUF506) gene family is widespread in many plant species. However, the basic information and knowledge of DUF506 genes are still lacking. The only functionally characterized DUF506 gene, At3g25240/RXR1/Repressor of excessive root hair growth1, which is highly induced by Pstress viaPHR1/PHL1-dependent pathway, is demonstrated to repress RH elongation. Overexpression of its closest homolog (Bdi2g58590) in Brachypodium confers the identical short RH phenotype (Ying, Blancaflor, Liao, \& Scheible, 2021). This intriguing trait promotes us to further investigate the P-response of other Arabidopsis DUF506 genes.

Here, additional P-inducible DUF506 genes are identified from public transcriptome database and their expression are validated by qRT-PCR. One of them, At1g62420/RXR3, is selected for comprehensively functional investigation. Our current results manifested that RXR3, differs from RXR1, is a novel root hair growth repressor through interaction with various cytosolic calmodulins.

\section{MATERIALS AND METHODS}

Plant material, growth and treatment

Arabidopsis thaliana (ecotype Col-0) seeds were sterilized and placed on half strength Murashige and Skoog (MS) solid medium according to (Ying et al., 2021). Petri dishes were stratified for 3 days at $4{ }^{\circ} \mathrm{C}$ in the dark before transfer to growth chamber $\left(120 \mathrm{\mu mol}^{-2} \mathrm{~s}^{-1}\right.$ light intensity, $22^{\circ} \mathrm{C} / 20{ }^{\circ} \mathrm{C}$, 16-h-light and 8-hdark cycle). For Phosphate-deprived (-P) treatment, the experiments were performed as described previously (MORCUENDE et al., 2007). In brief, Arabidopsis seedlings were germinated and grown in half strength MS liquid medium, and then transferred into -P liquid culture on day 7 . Shoot and root tissues were respectively harvested on day 9 or as indicated, then quickly frozen in liquid $\mathrm{N}_{2}$, and stored in $-80{ }^{\circ} \mathrm{C}$ freezer for further study. The rxr3-1 (Salk_016908), rxr3-2 (Salk_078603), cam7 (CS873256), and arf7 arf19 (CS24629) T-DNA insertion mutant lines were obtained from Arabidopsis Biological Resource Center (ABRC). The rsl4 mutant was provided by Dr. Elison Blancaflor (Noble Research Institute). Homozygous mutant plants were identified or validated according to the of SiGnAL database protocol (http://signal.salk.edu/).

Quantitative reverse transcription PCR ( $q R T-P C R)$

Total RNA was extracted using RNeasy ${ }^{\circledR}$ plant mini kit (QIAGEN) and genomic DNA was removed using 
Turbo DNA-free kit (Invitrogen) following the manufacturer's instructions. RNA (1 $\mu \mathrm{g})$ was converted into cDNA using SuperScript III Reverse Transcriptase (Invitrogen) according to the manufacture's instruction. Quantitative PCR was performed using QuantStudio 7 Flex Real-Time PCR System (Invitrogen) and KiCqStart ${ }^{\circledR}$ SYBR ${ }^{\circledR}$ Green qPCR ReadyMix(Millipore Sigma). The reaction procedures were executed as described previously (Ying et al., 2021). The Arabidopsis GAPDH(At1g13440) gene was used as an internal control (Czechowski, Stitt, Altmann, Udvardi, \& Scheible, 2005). All the experiments were repeated at least three times using cDNAs prepared from two biological replicates. Sequences of primers used in the study are listed in Supplemental Table 1.

Measurement of root hair length.

Seedlings were germinated vertically for 3 days on half strength MS solid medium containing $0.4 \%$ Gelzan CM (Millipore Sigma), and then transferred to plates containing either $0(-\mathrm{P})$ or $675 \mu \mathrm{M}(+\mathrm{P})$ phosphate, and $1 \mu \mathrm{M}$ synthesized AtRALF1 peptide (49 amino acids, Pepscan) respectively. Three days after transfer, root hair images were captured using a Nikon SMZ1500 stereo microscope. Root hair length was determined by measuring at least 700 root hairs located between 2 to $6 \mathrm{~mm}$ from the tip of the primary root in 10 individual plants from each line. Statistical analysis was performed as described previously (Ying et al., 2021).

\section{Generation of transformants (Overexpressor and complementation lines)}

For generation of $R X R 3$ gene overexpressing lines, full-length of coding region was amplified from Arabidopsis seedling cDNA using Phusion ${ }^{\circledR}$ High-Fidelity DNA polymerase (NEB) and cloned into GATEWAY ${ }^{\circledR}$ entry vector $\mathrm{pENTR} / \mathrm{SD} / \mathrm{D}-\mathrm{TOPO}^{(\mathrm{r})}$ (Invitrogen). Sequencing-confirmed vector was used to recombine into destination vector pMDC32 (Curtis \& Grossniklaus, 2003). For histochemical GUS analysis, a 1,300-bp upstream (from start codon) fragment of RXR3gene was amplified from Arabidopsis genomic DNA and subsequently cloned into the vector pBGWFS7 (Karimi, Inze, \& Depicker, 2002). For complementation analysis in the rxr2-1 mutant background, the promoter fragment of the $R X R 3$ gene and 852-bp coding region was respectively amplified, and subsequently constructed into destination vector pMU64 as described previously (Ying et al., 2021). For ChIP assay, the promoter fragment and full-length coding region of RSL4 gene was amplified from Arabidopsis seedling genomic DNA or cDNA, and eventually recombined into pMU64 vector, resulting in the proRSL4::RSL4-GFP construct. For calcium oscillation analysis, the proUBQ10::G-CAMP3GFP construct was transformed into individual rxr3 mutant lines (Kwon, Sparks, Liao, \& Blancaflor, 2018).

All constructs were introduced into Agrobacterium tumefaciensstrain GV3101 using a freeze-thaw procedure and were transformed into Arabidopsis by floral dipping (X. Zhang, Henriques, Lin, Niu, \& Chua, 2006). Transformants were selected on half strength MS agar $(0.8 \% \mathrm{w} / \mathrm{v})$ medium containing $25 \mu \mathrm{g} \mathrm{mL} \mathrm{m}^{-1}$ hygromycin (HYG, Omega Scientific).

\section{Western blotting}

For detection of GFP fusion protein in RXR3 complementation plants, total protein was extracted as described previously (Ying et al., 2021). Equal amount (30 $\mu \mathrm{g})$ of each sample was separated by SDS-PAGE, transferred into Immobilon-P PVDF membrane (0.45 $\mu \mathrm{M}$, Millipore Sigma), and probed with 1/5000-diluted monoclonal mouse anti-GFP horseradish peroxidase (HRP)-conjugated antibody (MiltenyiBiotec). Chemiluminescence detection was performed with ECL Prime Western Blotting System (Millipore Sigma) and UVP ChemStudio imager (Analytik Jena, US).

\section{Histochemical GUS staining and GFP imaging}

The histochemical GUS activity analysis was performed as previously described (Jefferson, Kavanagh, \& Bevan, 1987). Briefly, seedlings were incubated in a GUS staining solution containing $100 \mathrm{mM}$ sodium phosphate (pH 7.0), $1 \mathrm{mM}$ EDTA, 0.05\% Triton X-100, $1 \mathrm{mM}$ potassium ferricyanide/ferrocyanide, and 0.5 $\mathrm{mg} \mathrm{mL}{ }^{-1} \mathrm{X}$-glucuronide (Goldbio) at $37^{\circ} \mathrm{C}$ for 1 to $3 \mathrm{hr}$. Then samples were gradually cleared in $30 \%, 50 \%$, $70 \%$ and $100 \%$ (v/v) ethanol for 30 min. Images were acquired using Nikon SMZ1500 stereomicroscope. GFP 
fluorescence of the proRXR3::RXR3-GFP or proRSL4::RSL4-GFPcomplementation plants was imaged with Leica TCS SP8 confocal laser-scanning microscope (Ex: $488 \mathrm{~nm}$; Em $507 \mathrm{~nm}$ ).

Chromatin immunoprecipitation (ChIP) assay

The root tissue of RSL4-GFP complementation seedlings, which were grown vertically on half strength MS agar $(0.8 \% \mathrm{w} / \mathrm{v})$ medium for 12 day, were harvested, cross-linked, and stored in $-80{ }^{\circ} \mathrm{C}$ for further steps. ChIP assay was conducted as described previously (Haring et al., 2007; Nelson, Denisenko, \& Bomsztyk, 2006; Saleh, Alvarez-Venegas, \& Avramova, 2008).

\section{Calmodulin binding assay}

For heterologous expression of $R X R 3$, its full-length (FL) and N-terminally ( $\Delta 1-22$, residues 1 to 22 , including CaM Binding Domain/CaMBD) truncated cDNA fragment was respectively subcloned into pMAL-c5X vector (NEB) carrying an N-terminal Maltose Binding Protein (MBP) tag. For recombinant protein production, constructs were separately introduced into the Escherichia coli(NEB Express Competent cells C2523H). The purification was performed using amylose resin (NEB) according to the manufacture's instruction. Protein concentration was quantified using Piercerapid gold BCA protein assay kit (Thermo Fisher Scientific), and finally stored in $-80^{\circ} \mathrm{C}$ freezer for further study.

CaM binding assay was carried out according to previous study with small modification (Kato, Aoyama, \& Maeshima, 2013). Briefly, recombinant MBP-RXR $3^{\mathrm{FL}}$ or MBP-RXR3 ${ }^{\Delta 1-22}$ protein $(2 \mu \mathrm{g})$ were incubated with $25 \mu \mathrm{l} \mathrm{CaM-agrose} \mathrm{(Millipore} \mathrm{Sigma)} \mathrm{in} \mathrm{the} \mathrm{buffer} \mathrm{containing} 20 \mathrm{mM}$ Tris (pH 7.0), $150 \mathrm{mM} \mathrm{NaCl}$, and $0.5 \mathrm{mM} \mathrm{CaCl}_{2}$ or $2 \mathrm{mM}$ EGTA for $1 \mathrm{hr}$ at $4^{\circ} \mathrm{C}$. Then, the supernatant was discarded by centrifugation at $1470 \mathrm{~g}$ for $2 \mathrm{~min}$. After three times washing with the same buffer, the bound protein was finally disassociated by incubation with $2 \times$ SDS-PAGE protein sample buffer (Millipore Sigma). Samples were separated and transferred as described previously. Western blotting was performed using 1/5000-diluted monoclonal mouse anti-MBP-HRP antibody (MiltenyiBiotec).

Bimolecular fluorescence complementation (BiFC) assay

The BiFC analysis was performed as previously described (Kudla \& Bock, 2016; Waadt, Schlücking, Schroeder, \& Kudla, 2014). Briefly, RXR3 was respectively fused to the N-terminal EYFP in the pSITE-nEYFP or pCAMBIA1305-CFP (gift by Dr. Elison Blancaflor) vector, whereas various Arabidopsis CaM genes were fused with the C-terminal part of EYFP in pSITE-cEYFP. These different vector pairs were co-transformed into $N$. benthamiana through Agro-infiltration. Forty-eight hours after infiltration, YFP or CFP fluorescence was detected using a Leica TCS SP8 confocal laser-scanning microscope (CFP, Ex: 405 nm; Em: 485 nm; YFP, Ex: 514 nm; Em: 527 nm).

\section{Measurement of phosphate content}

The P-content measurement was conducted as described previously (Carter \& Karl, 1982) with minor modification. In brief, $50 \mathrm{mg}$ of Arabidopsis ground tissue was thoroughly resuspended in $600 \mu \mathrm{L}$ of MilliQ water. After centrifugation at $13000 \mathrm{~g}$ for $2 \mathrm{~min}$, the clarified supernatant was used to quantify the phosphate content. Mixture of $20 \mu \mathrm{L}$ of supernatant, $90 \mu \mathrm{L}$ of $1 \mathrm{~N} \mathrm{HCl}$, and $90 \mu \mathrm{L}$ of malachite green dye solution $(0.042 \%, \mathrm{w} / \mathrm{v})$ was incubated at room temperature for $5 \mathrm{~min}$, then the absorbance at $630 \mathrm{~nm}$ was recorded by spectrophotometer. The reading values were calibrated by the standard curve created by using various concentration of $\mathrm{K}_{2} \mathrm{HPO}_{4}$. All the measurements were repeated at least three times with representative results shown in the figures.

Statistical analysis

Statistical analyses and plotting were performed with GraphPad Prism 9.0 (GraphPad Software, Inc., San Diego, CA).

Accession Numbers 
Sequence data from this article can be found in the Phytozome 12 online genomic resource (https://phytozome.jgi.doe.gov/pz/portal.html) under the following accession numbers:AtRXR1 (At3g25240), AtRabD2c/AtRXR2 (At4g17530), AtRXR3 (At1g62420), Arabidopsis DUF506gene family members (At1g12030, At1g77145, At1g77160, At2g20670, At2g38820, At2g39650, At3g07350, At3g22970, At3g54550, At4g14620 and At4g32480), AtCAM1 (At5g37780), AtCAM2 (At2g41110), AtCAM6 (At5g21274), AtCAM7 (At3g43810), AtCNGC14 (At2g24610), AtRALF1 (At1g02900), and

AtEXPA7 (At1g12560).

\section{RESULTS}

Expression profile of Arabidopsis DUF506 gene family under P-limitation

To functionally characterize AtDUF506 genes under nutrient deprivation, we firstly examined their expression patterns from public RNA-seq database (i.e. Genevestigator), and found four additional DUF506 genes (e.g. At1g62420 , At2g20670 , At3g07350 , andAt4g32480) were induced by P-limitation. Next, we investigated the transcript changes of AtDUF506 genes in response to P-deprivation using qRT-PCR (Fig. 1). Similar to RXR1,At3g07350 and At4g32480 were significantly upregulated in both shoot and root tissue, whereas At1g62420 and At2g20670 respectively exhibited root or shoot-specific induction when exposed to P-stress. Owing to their high P-induction, we decided to detect the tissue-specific expression of At3g07350 and At1g62420 via GUS staining. As shown in Fig. S1, At3g07350gene expressed slightly in vascular bundles and lateral root tips under $+\mathrm{P}$ condition. Conversely, it was strongly induced throughout the entire seedling (e.g. cotyledon, primary/lateral root, root tips) after cultured in $-\mathrm{P}$ liquid medium for $24 \mathrm{hr}$. Meanwhile, At1g62420 was mainly visualized in primary rather than lateral root, such as vascular bundle, root tip and root hairs. In contrast, no distinguishable signals were detected in P-stressed shoot and P-sufficient cultured seedlings (Fig. 2B). These results suggested that Arabidopsis DUF506 genes broadly participated in P-stress response, and warranted to comprehensively demonstrate their roles by further study.

Transcriptional characterization of P-inducible At1g62420 gene

To investigate the specificity of P-inducible expression, time course experiment was employed to monitor the transcript changes of At1g62420 gene during P-starvation. The transcript was unchanged for $6 \mathrm{hr}$, and reached the peak at $24 \mathrm{hr}$ (Fig. 2A). After $3 \mathrm{hr}$ of P re-supplement, expression level of At1g62420 quickly resumed to regular level, indicating a direct response to the plant P-status.

PHOSPHATE STARVATION RESPONSE1 (PHR1) and its homolog PHR1-LIKE (PHL1) are the critical TFs of plant P-response (Bustos et al., 2010; NILSSON, MÜLLER, \& NIELSEN, 2007; Rubio et al., 2001). RXR1 was ascertained to be transcriptionally activated by PHR1 and PHL1 (Ying et al., 2021), we were interested to examine whether At1g62420 is regulated by these two TFs. To test this, we firstly looked for PHR1 binding sequences (P1BS) in its promoter region, but none was found. Next, we investigated the transcript changes of At1g62420 inphr1 phl1 double mutant to P treatment. In phr1 phl1 double mutant, At1g62420 exhibited identical expression pattern when compared to wild type, especially in root tissue (Fig. 2C), indicating it responds to P stress independently of PHR1 and PHL1.

Under P-limitation, the concentration of endogenous auxin is significantly accumulated and re-distributed in various organs, that leading to altered root system architecture (RSA). Therefore, we looked into the expression changes of At1g62420 to auxin treatment. Notably, the transcriptional inductions by auxin were comparable to those of P-stress (Fig. 2D), implying that auxin, not P status, directly modulated the transcription of At1g62420 .

Overexpression of At1g62420 represses root hair growth

To elucidate the biological function of At1962420 in plant, we firstly generated the overexpression lines. Two independent homozygous transgenic lines (e.g. OX-5 and OX-12) exhibited significant short ( $22 \%) \mathrm{RH}$ length compared to that of wild type (e.g. $+/-\mathrm{P}, 0.189 \pm 0.003 / 0.242 \pm 0.005 \mathrm{~mm}[\mathrm{OX}-12]$ versus $0.237 \pm$ $0.003 / 0.308 \pm 0.004 \mathrm{~mm}[\mathrm{WT}]$ ), when grown on P-sufficient or -deficient medium (Fig. 3B and C). Due to 
high phenotypic similarity toRXR1, At1g62420 was denoted as $R X R 3$ afterward. Secondly, we identified two independent loss-of-function mutants in ABRC, rxr3-1 (SALK_016908) and rxr3-2 (SALK_078603, Fig. $3 \mathrm{~A})$. The results of qRT-PCR analysis indicated that $R X R 3$ was undetectable in these mutants. RH length of mutants was longer than wild type (e.g. ${ }^{\sim} 41 \%$, rxr $3-1,-\mathrm{P}$ ) and overexpressors, in regardless of P-status (e.g. +/-P, $0.270 \pm 0.005 / 0.433 \pm 0.007 \mathrm{~mm}[$ rxr3-1 ]; $0.299 \pm 0.005 / 0.437 \pm 0.007 \mathrm{~mm}$ [rxr3-2 ], Fig. 3B and $\mathrm{C})$.

To ascertain the RH traits were tightly related to $R X R 3$ dysfunction, we introduced endogenous promoter driven RXR3-GFPconstruct in rxr3-1 mutant. GFP signal was detected from two independent complementation lines via Western blotting, implying that RXR3 was correctly transcribed and translated in vivo (Fig. 3F). Moreover, the RXR3-GFP mainly presented in root epidermal cells and root hair tips (Fig. 3G), hinted the close relationship between RXR3 and RH growth. RH lengths of complementation lines were similar to that of wild type, particularly under P-deficiency, indicating that $R X R 3$ appropriately rescued the abnormal phenotype of $r x r 3-1$ mutant (e.g. +/-P, $0.265 \pm 0.004 / 0.362 \pm 0.007 \mathrm{~mm}$ [Comp\#1] versus $0.233 \pm$ $0.004 / 0.327 \pm 0.006 \mathrm{~mm}[\mathrm{WT}]$, Fig. 3D and E).

Considering the similar inhibitory function of root hair elongation between RXR1 and RXR3, we generated the rxr1 rxr3-1 double mutant to validate their functional redundancy (Fig. 4). Under the P-replete condition, root hair length of double mutant $(0.360 \pm 0.005 \mathrm{~mm})$ was similar to $\operatorname{rxr} 1$ mutant $(0.366 \pm 0.006 \mathrm{~mm})$, but significantly longer than rxr3-1 mutant $(0.309 \pm 0.005 \mathrm{~mm})$ and wild type $(0.256 \pm 0.004 \mathrm{~mm})$. Under P-deficient condition, the double mutant $(0.456 \pm 0.007 \mathrm{~mm})$ exhibited slightly additive effects $(P<0.01)$ in relative to each single mutant $(r x r 1,0.421 \pm 0.007 \mathrm{~mm} ; r x r 3-1,0.430 \pm 0.006 \mathrm{~mm})$ or wild type (0.319 $\pm 0.005 \mathrm{~mm})$, suggesting that RXR3 inhibited RH growth possibly through separate pathway other than RXR1.

RHs are important for P-uptake, and as a result, the plant biomass increased accordingly (Crombez et al., 2019; Singh Gahoonia \& Nielsen, 2004). Unsurprisingly, rxr3-1 mutant exhibited distinguishable difference to wild type, regarding endogenous P-content or biomass (Fig. 3H, I and S3). In summary, these results suggested that RXR3 repressed RH elongation without interfering plant growth.

\section{RSL4 binds to the RXR3 promoter}

RSL4 has been demonstrated as a critical TF that dominated RH initiation and elongation (see Introduction). Thus, we investigated whether RXR3 participated in the RSL4-mediated regulatory pathway. According to previous reported consensus sequences (Hwang et al., 2017), four of RHE sites were identified in RXR3 promoter region (Fig.5A). Additionally, the P-inducible expression of $R X R 3$ was reversed in $r s l 4$ mutant (Fig.5B). Therefore, we presumed that RSL4 might directly bind to these RHSs to regulate RXR3expression. To test our hypothesis, we firstly generated the RSL4 complementation line that expressed its endogenous promoter drivenRSL4-GFP in rsl4 mutant. Nucleus-localized GFP signal was observed in root, indicated that RSL4-GFP fused protein was succeeded in rescuing the loss-of-function phenotype of rsl4 mutant (Fig.5D). Then, we conducted ChIP analysis to examine the interaction between RSL4 and RHE motifs in RXR3 promoter. EXPA7 is well-known RSL4 positive regulated gene (Yi et al., 2010), therefore, we employed it as positive control of ChIP assay (Fig. S4). As shown in Fig.5C, RSL4 interacted with the probe 1 and 2, which contains the identified RHE motifs, whereas no interaction was observed between RSL4 and probe 3 . These results suggested that RSL4 could directly bind to RHEs within the RXR3 promoter.

\section{AtRALF1 activates RXR3 expression}

Recently, Zhu and colleagues (Zhu et al., 2020) reviewed that RALF1 and receptor FERONIA (FER) act central roles in RH growth, through translational regulation RSL4 protein by phosphorylated eIF4E1. Thus, we thought that RALF1 could possibly activate the expression of RXR3 via RSL4. Unsurprisingly, RH growths were highly promoted in wild type and rxr3-1 mutant after RALF1 treatment (Fig.6A). Also, transcript level of RXR3 in wild type, but not in $r s l 4$, increased in response to RALF1 (Fig.6B and C). Lastly, we examined whether RALF1 affected the subcellular localization of RXR3. Five-day-old of $R X R 3$ complementation seedlings were incubated with half strength MS liquid medium supplemented $1 \mu \mathrm{M}$ 
RALF1 for 6 hr. In Fig.6D, RXR3 exhibited the same pattern as it responding to P-stress, which was mainly presented in root epidermis. The results implied that AtRALF1 induced RXR3 expression via RSL4.

\section{RXR3 interacts with Calmodulins}

Previously, no featured motifs other than DUF506 domain was found in RXR1, whereas in the current study, one N-terminal CaMBD was identified in between amino acids 11 and 22 (motif 1-12, [FILVW] $x x x x x x x x x x[F I L V W]$ ) of RXR3. To determine whether RXR3 could interact with CaMs, we heterogeneously expressed and purified the MBP-tagged recombinant RXR3 protein (MBP-RXR3 ${ }^{\mathrm{FL}}$ ), as well as the N-terminal truncated mutant (MBP-RXR3 ${ }^{\Delta 1-22}$, Fig.7A and Fig. S5). Both of recombinant proteins were individually incubated with CaM-agrose in the presence or absence of $\mathrm{Ca}^{2+}$. The bound protein was dissociated, subjected to SDS-PAGE, and detected by immunoblotting with antibodies against MBP tag. As shown in Fig.7B, RXR3 ${ }^{\mathrm{FL}}$ bound to CaM-agrose in the presence of $\mathrm{Ca}^{2+}$, but not in the presence of EGTA $\left(\mathrm{Ca}^{2+}\right.$ chelator). Meanwhile, RXR3 ${ }^{\Delta 1-22}$ did not bind to CaM-agrose, in regardless of $\mathrm{Ca}^{2+}$ status. These results indicated that the $\mathrm{N}$-terminal $\mathrm{CaMBD}$ of RXR3 was critical and sufficient for binding to $\mathrm{Ca}^{2+}{ }_{-} \mathrm{CaM}$ complex.

To validate the interaction between RXR3 and CaMs in vivo, we further conducted the BiFC assay by co-infiltration to $N$. benthamiana leave. In Arabidopsis, four isoforms (e.g. CaM1/4, CaM2/3/5, CaM6 and CaM7) which derived from seven of CaM encoding genes have been identified and studied (Bender \& Snedden, 2013; Zielinski, 1998). We selected four different CaM genes (i.e. CaM1 ,CaM2 , CaM6 and CaM7 ) that represented all isoforms to carry on the BiFC assay. In Fig.7C, strong cytosolic yellow fluorescence signal was observed in each combination, illustrating that RXR3 interacted with various CaMs in vivo . Conversely, no signal was detected between CaM7 and RXR1, which served as negative control. Overall, these results demonstrated that RXR3 physically interacted with CaMs.

RXR3 influences on root hair $\left[\mathrm{Ca}^{2+}\right]_{\text {cyt }}$ oscillation

Calmodulins function as versatile $\mathrm{Ca}^{2+}$-binding proteins that regulate the activity of numerous effectors (e.g. CNGCs) in $\mathrm{Ca}^{2+}$ signaling. It is reported that CaM7 bound with CNGC14 to regulate RH polar growth by controlling intrinsic $\mathrm{Ca}^{2+}$ status (Zeb et al., 2020). Thus, we investigated the effect of RXR3 protein on the interaction of CaM7 with CNGC14. When RXR3-CFP was co-expressed with CaM7-nEYFP and CNGC14-cEYFP in tobacco, the yellow fluorescence was not noticeably affected (Fig.8A and B). RXR3 was not only co-localized with CNCG14-CaM7 complex in cytosol, but also presenting in nucleus (Arrow, Fig.8B). These results indicated that RXR3 did not interfere in the interaction of CaM7 with CNGC14, and might conduct other functions in nucleus. Furthermore, we expressed GCaMP-GFP construct in wild type, rxr3-1 and rxr3-2 mutant respectively, to quantitatively examined whether RXR3 influences the apical $\left[\mathrm{Ca}^{2+}\right]_{\text {cyt }}$ oscillation in root hair. Both rxr3 mutants (rxr3-1, $0.0517 \pm 0.0027 \mathrm{~Hz} ;$ rxr3-2, $0.0542 \pm 0.0020$ $\mathrm{Hz})$ exhibited $\sim 20 \%$ higher frequency of $\left[\mathrm{Ca}^{2+}\right]_{\text {cyt }}$ oscillations compared to that in wild type $(0.0399 \pm 0.0009$ $\mathrm{Hz}$ ), indicating that RXR3 repressed $\mathrm{RH}$ growth possibly by regulating $\left[\mathrm{Ca}^{2+}\right]_{\text {cyt }}$ oscillations (Fig. $8 \mathrm{C}$ and D).

Overexpression of $\mathrm{CaM7}$ dramatically represses RH growth by inhibition of CNGC14 activity (Zeb et al., 2020). Here, we found thatcam 7 mutant exhibited longer RH length in relative to wild type. Additionally, when constitutively overexpressing $R X R 3^{F L}$ in $c a m 7$ mutant, the long $R H$ phenotype was not restored (Fig. S7). These results suggested that RXR3 affected RH growth and $\left[\mathrm{Ca}^{2+}\right]_{\text {cyt }}$ oscillation through interaction of CaM7.

\section{DISCUSSION}

Domain of unknown function (DUF) is a conserved protein domain that has not been functionally characterized or annotated. Recently, we demonstrated that P-inducible RXR1, which belongs to DUF506 family, negatively regulates RH growth through interacting with small GTPase RabD2c/RXR2 (Ying et al., 2021). Here, we have expanded our study with identification and characterization of four other P-inducible DUF506 genes in Arabidopsis. 
Based on the relatively high P-induction expression, we selected two of the AtDUF506 genes for in-depth characterization.At3g07350, which is the duplicated pair of $R X R 1$, was previously recognized as uncharacterized and putative root hair specific gene with two RHE in its promoter region (Won et al., 2009). At that time, a set of 37 Arabidopsis RHS genes were identified through genome-wide RHE motif screening in gene promoter region together with transcriptome analysis. Meanwhile, 65\% (24/37) of RHS genes were experimentally validated their relationship of RH morphology (Won et al., 2009). For instance, one cell-wall associated receptor kinase encoding gene, RHS10 (At1g70460), was demonstrated to be activated by RSL4 and inhibit RH growth through suppression of ROS accumulation and RNA metabolism (Hwang et al., 2017; Hwang, Lee, Lee, \& Cho, 2016). Here, GUS staining results discovered that under P-stress At3g07350 was highly induced in roots, especially root tips and root hairs (Fig. S1). The root tip focused expression pattern differs from $R X R 1$, which mostly presents in vascular bundle but is undetectable in primary root tip. Moreover, expression analysis revealed that At3g07350 in root was induced by auxin (Fig. S2A), suggesting its involvement in auxin-dependent regulatory pathway. It is reported that TAA1, which expresses at root apex and manages auxin synthesis, is up-regulated under low-P condition that led to activation of AUX1-dependent shootward auxin transport pathway accompanied with $R S L 4$ transcript up-regulation, and eventually root hairs elongation (Bhosale et al., 2018; Datta et al., 2015). It is noteworthy that At3g07350 is the only RHS gene showing up-regulation (i.e. 2-fold) in rhd6 mutant background (Won et al., 2009), suggesting that it might be directly or indirectly suppressed by RHD6 in root epidermis. At3g07350 was not included in the set of 208 core root epidermal genes in Arabidopsis (Bruex et al., 2012), possibly owing to the paucity of transcript under normal condition, or irrelevance of RH initiation. Collectively, we speculated that At3g07350 acted a role in aforementioned auxin-mediated $\mathrm{P}$-signal pathway, different from $R X R 1$. In future, additional experiments (e.g. characterization of its overexpressor andloss-of-function mutants) are necessary to discover the functions of At3g07350 in respect to P-signaling and RH development.

In order to investigate the biological function of the third P-inducible DUF506 gene, we performed additional genetic, molecular, biochemical and cytological experiments (Fig. 2 to 8). Similar expression pattern (e.g. tissue-specific and P-response) as well as GUS staining results (i.e. root hair) between $R X R 1$ and $R X R 3$ , implicate the possibility of functional redundancy. Overexpression of $R X R 3$ significantly limits the RH length, whereas the knock-out mutants exhibit opposite phenotype (Fig. 3), that remind us the inhibitory role of RXR1 to RH elongation. Despite both of RXR1 and RXR3 shares highly similar molecular and functional features, our studies reveal that the regulatory mechanism is distinct from each other. For instance, $R X R 1$ magnificently responds to P-stress (i.e. >1000-fold up-regulation) through PHR1/PHL1-dependent pathway, and independently of auxin (Ying et al., 2021). In contrast, $R X R 3$ in root functions in PHR1/PHL1independent pathway and is induced by auxin (Fig. 2). Furthermore, the $\operatorname{rxr} 1$ rxr3-1 double mutant exhibits extra longer root hair when compared to each single mutant (Fig. 4), implicated the functional divergency between RXR1 and RXR3.

$R S L 4$ is moderately up-regulated by P-limitation, and promotes $\mathrm{RH}$ growth accordingly (Bhosale et al., 2018; Datta et al., 2015; Yi et al., 2010). Our results demonstrate that RSL4 TF binds to RHE motifs in $R X R 3$ promoter, resulting in its transcript accumulation (Fig.5). Zhu et al. (2020) summarized an autocrine signaling pathway that involved RALF1-FERONIA-RSL4 to synergistically regulation RH size. RALF1 was proved to promote the expression of $R S L 4$, which consequently caused the accumulation of $R X R 3$ transcript (Fig.6). Thus, we could conclude that RXR3 participates in auxin and RSL4-dependent signaling pathway, in order to manage P-induced root hair elongation (see Fig.9).

Beside of the RHE motifs, three copies of auxin responsive element (i.e. AACGAC) were found in $R X R 3$ promoter (i.e. respectively localized in $-116,-184$ and -454 bp upstream of start codon) as well. Previously, ChIP assay and genetic evidences demonstrated that both RSL2 and RSL4 are transcriptionally activated by auxin response factors (ARFs) (Bhosale et al., 2018; S. Mangano et al., 2017). Under P-limitation, high auxin level induced the expression of severalARFs (e.g. ARF5, 7, 8 and 19 ), leading to the activation of $R S L 4$ and possible RSL2. On the other hand, when Pi is sufficient in soil, endogenous auxin activates RSL2 expression, but not RSL4, to regulate ROS production, and $\mathrm{RH}$ growth in subsequent (Bhosale et al., 2018; Silvina Mangano et al., 2018). qRT-PCR analysis results indicated that transcript of RXR3was 
moderately reduced in arf7 arf19 double mutant, in regardless of P-status (Fig. S2B). Therefore, we propose that RXR3 might not only be managed directly by RSL4, but also ARFs, especially under P-deficient condition, to coordinatively control RH growth (Fig.9).

$\mathrm{Ca}^{2+}$, as indispensable macronutrient and second messenger molecule, could associate with various $\mathrm{Ca}^{2+}$ binding protein (e.g. CaM and CDPK) by EF-hand motifs, to vastly modulate many biological processes in plants (Hepler, 2005; Himschoot, Beeckman, Friml, \& Vanneste, 2015; Thor, 2019; Yuan, Jauregui, Du, Tanaka, \& Poovaiah, 2017). Accumulating evidences suggested that rapid and gradient oscillation of intracellular $\mathrm{Ca}^{2+}$ concentration $\left(\left[\mathrm{Ca}^{2+}\right]_{\text {cyt }}\right)$ was critical for sustained root hair polar tip growth (Bibikova, Zhigilei, \& Gilroy, 1997; W. Feng et al., 2018; Konrad, Wudick, \& Feijó, 2011; Monshausen, Messerli, \& Gilroy, 2008). Recently, CNGC14 is reported to be in charge of stabilizing the integrity of growing RH and supporting cell expansion as well, and prone to interact with CaM7, to inhibit RH tip growth (Brost et al., 2019; Zeb et al., 2020; S. Zhang et al., 2017). Except of RXR1, CaMBD has been identified in other members of Arabidopsis DUF506 family. Protein microarray analysis have demonstrated that At1g77145 interacted with several CaM or CaM-like (CML) proteins (Popescu et al., 2007). Here, we found that RXR3 could interact with various $\mathrm{Ca}^{2+}$-bound $\mathrm{CaMs}$ in vivo (Fig.7). When overexpressing $P \Xi P 3^{\Delta 1-22}$ in rxr3-2 mutant, it could not restore the long root hair phenotype (Fig. S6), suggested the CaMBD was critical for inhibitory function. Moreover, RXR3 was unable to interrupt the association between CaM7 and CNGC14 (Fig.8B). Similar to RXR3, overexpression of $\mathrm{CaM}^{7}$ gene conferred to shorter RH length (Zeb et al., 2020). The long RH phenotype of $\mathrm{cam}^{7}$ mutant could not be restored by constitutively expressing $R X R 3$ gene (Fig. S7), demonstrated that $\mathrm{CaM}^{7}$ was required for $R X R 3$ conducting its inhibitory roles. Considering the root hair tips focused localization (Fig. 3G and 6D), we hypothesized that, under P-limitation, RSL4-activated RXR3 might play as an escort or stabilizer, associates with and delivers $\mathrm{Ca}^{2+}$-bound CaM7 to plasma membrane, to simultaneously inhibit CNGC14 activation and RH growth (Fig.9).

Besides CNGC14, other CNGCs, have been demonstrated to be important regulators of root hair or pollen tube tip growth (Brost et al., 2019; Chang, Yan, Zhao, Wu, \& Yang, 2007; Gao et al., 2016; Pan et al., 2019; Tan et al., 2020; Tunc-Ozdemir et al., 2013; S. Zhang et al., 2017). Moreover, some of CNGCs could spontaneously form heteromeric complexes, such as CNGC9/14 and CNGC2/4, to conduct their regulatory roles (Brost et al., 2019; Chin, DeFalco, Moeder, \& Yoshioka, 2013; Yu et al., 2019). In contrast to CaM7-arrested CNGC14, the activity of other CNGCs could be positively regulated by CaM binding (e.g. CaM1 to CNGC12)(Dietrich, Moeder, \& Yoshioka, 2020; Jarratt-Barnham, Wang, Ning, \& Davies, 2021). These aforementioned studies have shed light on the diverse regulatory mechanism of CNGC. We have characterized the capacity of RXR3 interacting with different kinds of CaM isoforms, hint that RXR3, and perhaps other DUF506 proteins (e.g. At1g77145), are novel components in regulation of CaM-CNGCmediated $\mathrm{Ca}^{2+}$ dynamics and oscillation. Further research is required to explore DUF506-CaM interacting specificity and to understand the biological function of individual DUF506 proteins.

In an effort to assess the influences of $R X R 3$ on $\left[\mathrm{Ca}^{2+}\right]_{\text {cyt }}$ changes, we introduced GCaMP3-GFP construct to two individual rxr3 mutant lines, and recorded the $\left[\mathrm{Ca}^{2+}\right]_{\mathrm{cyt}}$ oscillation in root hair apex zone (Fig.8C and D). Frequency of $\left[\mathrm{Ca}^{2+}\right]_{\mathrm{cyt}}$ oscillation elevated about $20 \%$ in both $\operatorname{rxr} 3$ mutants compared to that of wild type, manifested that RXR3 negatively affected tip-focused $\left[\mathrm{Ca}^{2+}\right]_{\text {cyt }}$ gradients. Interestingly, the frequency of $\left[\mathrm{Ca}^{2+}\right]_{\text {cyt }}$ oscillation inrxr1 mutant showed the similar level $\left({ }^{\sim} 25 \%\right)$ of elevation (Ying et al., 2021). Therefore, we could not rule out the possibility of the interaction between RXR2 and RXR3, because of the widespread of conserved domain 3 in DUF506 family. On the other hand, these results confirmed the importance of elements outside of the conserved domain of DUF506 protein (Fig. S6).

While additional insights would be required to systematically illustrate the mechanism, evidence here supports a model in which RXR3 has inhibitory function that are essential for the maintenance of environmental stress induced RH growth (Fig.9). It remains to be determined as to whether and/or how RXR3 could connect with the other tip growth focused CNGCs. Our present evidence, together with previous studies, suggest that the RXR1 and RXR3 function as novel complements of P-inducible RH growth regulatory network, and could be considered as crop engineering targets to improve crop/forage resilience against rhizosphere 
nutrient deficiency.

\section{ACKNOWLEDGEMENT}

We thank to Dr. Elison Blancaflor for providing plasmids, rsl4mutant seeds and advice of operating microscopes, Fuqi Liao for measurement of root hair length and calcium oscillation, Sylvia Warner for technical assistance, and Dr. Marcus Griffins (Donald Danforth Plant Science Center) for proofreading and critical comments. The work was funded by the Noble Research Institute LLC.

\section{SUPPORTING INFORMATION}

Fig. S1 . GUS staining assay of the proAt3g07350::GUStransgenic line.

Fig. S2 . Expression analysis by qRT-PCR.

Fig. S3 . Phenotype of 10-day-old Arabidopsis seedlings (WT andrxr3-1) grown on $+\mathrm{P}$ or $-\mathrm{P}$ half strength MS solid medium.

Fig. S4 . RSL4 binds to the EXPA7 promoter.

Fig. S5 . Recombinant expression of MBP-RXR3 $3^{\mathrm{FL}}(75.2 \mathrm{kDa})$ and MBP-RXR3 $3^{\Delta 1-22}(72.7 \mathrm{kDa})$ protein.

Fig. S6 . Constitutive overexpression of CaMBD-truncated $R X R 3\left(\pi \rho \circ a ̊ M^{\mathrm{v}} 35 \Sigma:: P \Xi P 3^{\Delta 1-22}\right)$ in rxr3-2 mutant.

Fig. S7 . Constitutive overexpression of $R X R 3^{F L}\left(\right.$ proCaMV35S::RXR $\left.3^{F L}\right)$ in cam7 mutant.

Table S1 . Primer sequences used in this study.

\section{AUTHOR CONTRIBUTION}

S.Y. and W.-R. S. conceived and designed the research. S.Y. performed experimental work, analyzed data and wrote manuscript. W.-R.S. coordinated the project, and provided overall supervision.

\section{CONFLICTS OF INTEREST}

The authors declare no conflict of interest.

\section{REFERENCE}

Abarca, A., Franck, C. M., \& Zipfel, C. (2021). Family-wide evaluation of RAPID ALKALINIZATION FACTOR peptides. Plant Physiology . doi:10.1093/plphys/kiab308

Bari, R., Datt Pant, B., Stitt, M., \& Scheible, W.-R. (2006). PHO2, MicroRNA399, and PHR1 Define a Phosphate-Signaling Pathway in Plants.Plant Physiology, 141 (3), 988-999. doi:10.1104/pp.106.079707

Bates, T. R., \& Lynch, J. P. (2001). Root hairs confer a competitive advantage under low phosphorus availability. Plant and Soil, 236 (2), 243-250. doi:10.1023/A:1012791706800

Bender, K. W., \& Snedden, W. A. (2013). Calmodulin-related proteins step out from the shadow of their namesake. Plant Physiol, 163 (2), 486-495. doi:10.1104/pp.113.221069

Bhosale, R., Giri, J., Pandey, B. K., Giehl, R. F. H., Hartmann, A., Traini, R., . . . Swarup, R. (2018). A mechanistic framework for auxin dependent Arabidopsis root hair elongation to low external phosphate.Nature Communications, 9 (1), 1409. doi:10.1038/s41467-018-03851-3

Bibikova, T. N., Zhigilei, A., \& Gilroy, S. (1997). Root hair growth in Arabidopsis thaliana is directed by calcium and an endogenous polarity.Planta, 203 (4), 495-505. doi:10.1007/s004250050219

Brost, C., Studtrucker, T., Reimann, R., Denninger, P., Czekalla, J., Krebs, M., . . . Dietrich, P. (2019). Multiple cyclic nucleotide-gated channels coordinate calcium oscillations and polar growth of root hairs. The Plant Journal, 99 (5), 910-923. doi:https://doi.org/10.1111/tpj.14371 
Brown, L. K., George, T. S., Dupuy, L. X., \& White, P. J. (2013). A conceptual model of root hair ideotypes for future agricultural environments: what combination of traits should be targeted to cope with limited $\mathrm{P}$ availability? Annals of Botany, 112 (2), 317-330. doi:10.1093/aob/mcs231

Bruex, A., Kainkaryam, R. M., Wieckowski, Y., Kang, Y. H., Bernhardt, C., Xia, Y., . . . Schiefelbein, J. (2012). A Gene Regulatory Network for Root Epidermis Cell Differentiation in Arabidopsis. PLoS genetics, 8 (1), e1002446. doi:10.1371/journal.pgen.1002446

Bucher, M. (2007). Functional biology of plant phosphate uptake at root and mycorrhiza interfaces. New Phytologist, 173 (1), 11-26. doi:https://doi.org/10.1111/j.1469-8137.2006.01935.x

Bustos, R., Castrillo, G., Linhares, F., Puga, M. I., Rubio, V., Pérez-Pérez, J., . . Paz-Ares, J. (2010). A Central Regulatory System Largely Controls Transcriptional Activation and Repression Responses to Phosphate Starvation in Arabidopsis. PLoS genetics, 6 (9), e1001102. doi:10.1371/journal.pgen.1001102

Carter, S. G., \& Karl, D. W. (1982). Inorganic phosphate assay with malachite green: An improvement and evaluation. Journal of Biochemical and Biophysical Methods, 7 (1), 7-13. doi:https://doi.org/10.1016/0165022X(82)90031-8

Chang, F., Yan, A., Zhao, L.-N., Wu, W.-H., \& Yang, Z. (2007). A Putative Calcium-Permeable Cyclic Nucleotide-Gated Channel, CNGC18, Regulates Polarized Pollen Tube Growth. Journal of Integrative Plant Biology, 49 (8), 1261-1270. doi:https://doi.org/10.1111/j.1672-9072.2007.00524.x

Chin, K., DeFalco, T. A., Moeder, W., \& Yoshioka, K. (2013). The Arabidopsis cyclic nucleotide-gated ion channels AtCNGC2 and AtCNGC4 work in the same signaling pathway to regulate pathogen defense and floral transition. Plant Physiology, 163 (2), 611-624. doi:10.1104/pp.113.225680

Cho, H. T., \& Cosgrove, D. J. (2002). Regulation of root hair initiation and expansin gene expression in Arabidopsis. Plant Cell, 14 (12), 3237-3253. doi:10.1105/tpc.006437

Choi, H.-S., \& Cho, H.-T. (2019). Root hairs enhance Arabidopsis seedling survival upon soil disruption. Scientific Reports, 9 (1), 11181. doi:10.1038/s41598-019-47733-0

Crombez, H., Motte, H., \& Beeckman, T. (2019). Tackling Plant Phosphate Starvation by the Roots. Developmental Cell, 48 (5), 599-615. doi:https://doi.org/10.1016/j.devcel.2019.01.002

Curtis, M. D., \& Grossniklaus, U. (2003). A gateway cloning vector set for high-throughput functional analysis of genes in planta. Plant Physiol, 133 (2), 462-469. doi:10.1104/pp.103.027979

Czechowski, T., Stitt, M., Altmann, T., Udvardi, M. K., \& Scheible, W.-R. (2005). Genome-Wide Identification and Testing of Superior Reference Genes for Transcript Normalization in Arabidopsis. Plant Physiology, 139 (1), 5-17. doi:10.1104/pp.105.063743

Datta, S., Prescott, H., \& Dolan, L. (2015). Intensity of a pulse of RSL4 transcription factor synthesis determines Arabidopsis root hair cell size. Nat Plants, 1 , 15138. doi:10.1038/nplants.2015.138

Dietrich, P., Moeder, W., \& Yoshioka, K. (2020). Plant Cyclic Nucleotide-Gated Channels: New Insights on Their Functions and Regulation. Plant Physiology, 184 (1), 27-38. doi:10.1104/pp.20.00425

Feng, W., Kita, D., Peaucelle, A., Cartwright, H. N., Doan, V., Duan, Q., . . Dinneny, J. R. (2018). The FERONIA Receptor Kinase Maintains Cell-Wall Integrity during Salt Stress through $\mathrm{Ca}^{2+}$ Signaling. Current Biology, 28 (5), 666-675.e665. doi:https://doi.org/10.1016/j.cub.2018.01.023

Feng, Y., Xu, P., Li, B., Li, P., Wen, X., An, F., . . Guo, H. (2017). Ethylene promotes root hair growth through coordinated EIN3/EIL1 and RHD6/RSL1 activity in Arabidopsis. Proceedings of the National Academy of Sciences, 114 (52), 13834-13839. doi:10.1073/pnas.1711723115

Foreman, J., Demidchik, V., Bothwell, J. H. F., Mylona, P., Miedema, H., Torres, M. A., . . Dolan, L. (2003). 
Reactive oxygen species produced by NADPH oxidase regulate plant cell growth. Nature, 422 (6930), 442446. doi:10.1038/nature01485

Gao, Q. F., Gu, L. L., Wang, H. Q., Fei, C. F., Fang, X., Hussain, J., . . Wang, Y. F. (2016). Cyclic nucleotide-gated channel 18 is an essential $\mathrm{Ca}^{2+}$ channel in pollen tube tips for pollen tube guidance to ovules in Arabidopsis. Proc Natl Acad Sci U S A, 113 (11), 3096-3101. doi:10.1073/pnas.1524629113

Grierson, C., Nielsen, E., Ketelaarc, T., \& Schiefelbein, J. (2014). Root hairs. The arabidopsis book, 12 , e0172-e0172. doi:10.1199/tab.0172

Haling, R. E., Brown, L. K., Bengough, A. G., Young, I. M., Hallett, P. D., White, P. J., \& George, T. S. (2013). Root hairs improve root penetration, root-soil contact, and phosphorus acquisition in soils of different strength. Journal of Experimental Botany, 64 (12), 3711-3721. doi:10.1093/jxb/ert200

Hammond, J. P., Broadley, M. R., \& White, P. J. (2004). Genetic responses to phosphorus deficiency. Ann Bot, 94 (3), 323-332. doi:10.1093/aob/mch156

Han, X., Zhang, M., Yang, M., \& Hu, Y. (2020). Arabidopsis JAZ Proteins Interact with and Suppress RHD6 Transcription Factor to Regulate Jasmonate-Stimulated Root Hair Development. The Plant Cell, 32 (4), 1049-1062. doi:10.1105/tpc.19.00617

Haring, M., Offermann, S., Danker, T., Horst, I., Peterhansel, C., \& Stam, M. (2007). Chromatin immunoprecipitation: optimization, quantitative analysis and data normalization. Plant Methods, 3 (1), 11. doi:10.1186/1746-4811-3-11

Hepler, P. K. (2005). Calcium: a central regulator of plant growth and development. Plant Cell, 17 (8), 2142-2155. doi:10.1105/tpc. 105.032508

Himschoot, E., Beeckman, T., Friml, J., \& Vanneste, S. (2015). Calcium is an organizer of cell polarity in plants. Biochim Biophys Acta, 1853 (9), 2168-2172. doi:10.1016/j.bbamcr.2015.02.017

Hwang, Y., Choi, H.-S., Cho, H.-M., \& Cho, H.-T. (2017). Tracheophytes Contain Conserved Orthologs of a Basic Helix-Loop-Helix Transcription Factor That Modulate ROOT HAIR SPECIFIC Genes. The Plant Cell, 29 (1), 39-53. doi:10.1105/tpc.16.00732

Hwang, Y., Lee, H., Lee, Y.-S., \& Cho, H.-T. (2016). Cell wall-associated ROOT HAIR SPECIFIC 10, a proline-rich receptor-like kinase, is a negative modulator of Arabidopsis root hair growth. Journal of Experimental Botany, 67 (6), 2007-2022. doi:10.1093/jxb/erw031

Jarratt-Barnham, E., Wang, L., Ning, Y., \& Davies, J. M. (2021). The Complex Story of Plant Cyclic Nucleotide-Gated Channels.International Journal of Molecular Sciences, 22 (2), 874.

Jefferson, R. A., Kavanagh, T. A., \& Bevan, M. W. (1987). GUS fusions: beta-glucuronidase as a sensitive and versatile gene fusion marker in higher plants. The EMBO Journal, 6 (13), 3901-3907.

Kapulnik, Y., Delaux, P. M., Resnick, N., Mayzlish-Gati, E., Wininger, S., Bhattacharya, C., .. . Koltai, H. (2011). Strigolactones affect lateral root formation and root-hair elongation in Arabidopsis.Planta, 233 (1), 209-216. doi:10.1007/s00425-010-1310-y

Karimi, M., Inzé, D., \& Depicker, A. (2002). GATEWAY vectors for Agrobacterium-mediated plant transformation. Trends in Plant Science, 7 (5), 193-195. doi:https://doi.org/10.1016/S1360-1385(02)02251-3

Kato, M., Aoyama, T., \& Maeshima, M. (2013). The $\mathrm{Ca}^{2+}$-binding protein PCaP2 located on the plasma membrane is involved in root hair development as a possible signal transducer. The Plant Journal, 74 (4), 690-700. doi:https://doi.org/10.1111/tpj.12155

Kim, C. M., \& Dolan, L. (2016). ROOT HAIR DEFECTIVE SIX-LIKE Class I Genes Promote Root Hair Development in the Grass Brachypodium distachyon. PLoS genetics, 12 (8), e1006211. doi:10.1371/journal.pgen.1006211 
Kim, C. M., Han, C.-d., \& Dolan, L. (2017). RSL class I genes positively regulate root hair development in Oryza sativa. New Phytologist, 213 (1), 314-323. doi:https://doi.org/10.1111/nph.14160

Konrad, K. R., Wudick, M. M., \& Feijo, J. A. (2011). Calcium regulation of tip growth: new genes for old mechanisms. Current Opinion in Plant Biology, 14 (6), 721-730. doi:https://doi.org/10.1016/j.pbi.2011.09.005

Kudla, J., \& Bock, R. (2016). Lighting the Way to Protein-Protein Interactions: Recommendations on Best Practices for Bimolecular Fluorescence Complementation Analyses. The Plant Cell, 28 (5), 1002-1008. doi:10.1105/tpc.16.00043

Kwasniewski, M., Daszkowska-Golec, A., Janiak, A., Chwialkowska, K., Nowakowska, U., Sablok, G., \& Szarejko, I. (2016). Transcriptome analysis reveals the role of the root hairs as environmental sensors to maintain plant functions under water-deficiency conditions. Journal of Experimental Botany, 67 (4), 10791094. doi:10.1093/jxb/erv498

Kwon, T., Sparks, J. A., Liao, F., \& Blancaflor, E. B. (2018). ERULUS Is a Plasma Membrane-Localized Receptor-Like Kinase That Specifies Root Hair Growth by Maintaining Tip-Focused Cytoplasmic Calcium Oscillations. The Plant Cell, 30 (6), 1173-1177. doi:10.1105/tpc.18.00316

Lin, Q., Ohashi, Y., Kato, M., Tsuge, T., Gu, H., Qu, L.-J., \& Aoyama, T. (2015). GLABRA2 Directly Suppresses Basic Helix-Loop-Helix Transcription Factor Genes with Diverse Functions in Root Hair Development. The Plant Cell, 27 (10), 2894-2906. doi:10.1105/tpc.15.00607

Lynch, J. P. (2011). Root Phenes for Enhanced Soil Exploration and Phosphorus Acquisition: Tools for Future Crops. Plant Physiology, 156 (3), 1041-1049. doi:10.1104/pp.111.175414

Lynch, J. P. (2019). Root phenotypes for improved nutrient capture: an underexploited opportunity for global agriculture. New Phytologist, 223 (2), 548-564. doi:https://doi.org/10.1111/nph.15738

Ma, Z., Baskin, T. I., Brown, K. M., \& Lynch, J. P. (2003). Regulation of root elongation under phosphorus stress involves changes in ethylene responsiveness. Plant Physiology, 131 (3), 1381-1390. doi:10.1104/pp.012161

Mangano, S., Denita-Juarez, S. P., Choi, H. S., Marzol, E., Hwang, Y., Ranocha, P., . . . Estevez, J. M. (2017). Molecular link between auxin and ROS-mediated polar growth. Proc Natl Acad Sci U S A, 114 (20), 5289-5294. doi:10.1073/pnas.1701536114

Mangano, S., Denita-Juarez, S. P., Marzol, E., Borassi, C., \& Estevez, J. M. (2018). High Auxin and High Phosphate Impact on RSL2 Expression and ROS-Homeostasis Linked to Root Hair Growth in Arabidopsis thaliana.Frontiers in Plant Science, 9 (1164). doi:10.3389/fpls.2018.01164

Marin, M., Feeney, D. S., Brown, L. K., Naveed, M., Ruiz, S., Koebernick, N., . . . George, T. S. (2020). Significance of root hairs for plant performance under contrasting field conditions and water deficit. Annals of Botany . doi:10.1093/aob/mcaa181

Masucci, J. D., \& Schiefelbein, J. W. (1994). The rhd6 Mutation of Arabidopsis thaliana Alters RootHair Initiation through an Auxin- and Ethylene-Associated Process. Plant Physiology, 106 (4), 1335-1346. doi:10.1104/pp.106.4.1335

Monshausen, G. B., Messerli, M. A., \& Gilroy, S. (2008). Imaging of the Yellow Cameleon 3.6 Indicator Reveals That Elevations in Cytosolic $\mathrm{Ca}^{2+}$ Follow Oscillating Increases in Growth in Root Hairs of Arabidopsis. Plant Physiology, 147 (4), 1690-1698. doi:10.1104/pp.108.123638

MORCUENDE, R., BARI, R., GIBON, Y., ZHENG, W., PANT, B. D., BLASING, O., . . SCHEIBLE, W.R. (2007). Genome-wide reprogramming of metabolism and regulatory networks of Arabidopsis in response to phosphorus.Plant, Cell \& Environment, 30 (1), 85-112. doi:10.1111/j.1365-3040.2006.01608.x 
Nelson, J. D., Denisenko, O., \& Bomsztyk, K. (2006). Protocol for the fast chromatin immunoprecipitation (ChIP) method. Nat Protoc, 1 (1), 179-185. doi:10.1038/nprot.2006.27

NILSSON, L., MULLER, R., \& NIELSEN, T. H. (2007). Increased expression of the MYB-related transcription factor, PHR1, leads to enhanced phosphate uptake in Arabidopsis thaliana. Plant, Cell \& Environment, 30 (12), 1499-1512. doi:10.1111/j.1365-3040.2007.01734.x

Pan, Y., Chai, X., Gao, Q., Zhou, L., Zhang, S., Li, L., \& Luan, S. (2019). Dynamic Interactions of Plant CNGC Subunits and Calmodulins Drive Oscillatory $\mathrm{Ca}^{2+}$ Channel Activities. Dev Cell, 48 (5), 710-725.e715. doi:10.1016/j.devcel.2018.12.025

Parker, J. S., Cavell, A. C., Dolan, L., Roberts, K., \& Grierson, C. S. (2000). Genetic Interactions during Root Hair Morphogenesis in Arabidopsis. The Plant Cell, 12 (10), 1961-1974. doi:10.1105/tpc.12.10.1961

Pitts, R. J., Cernac, A., \& Estelle, M. (1998). Auxin and ethylene promote root hair elongation in Arabidopsis. The Plant Journal, 16 (5), 553-560. doi:10.1046/j.1365-313x.1998.00321.x

Plaxton, W. C., \& Tran, H. T. (2011). Metabolic Adaptations of Phosphate-Starved Plants. Plant Physiology, 156 (3), 1006-1015. doi:10.1104/pp.111.175281

Popescu, S. C., Popescu, G. V., Bachan, S., Zhang, Z., Seay, M., Gerstein, M., . . . Dinesh-Kumar, S. P. (2007). Differential binding of calmodulin-related proteins to their targets revealed through high-density Arabidopsis protein microarrays. Proceedings of the National Academy of Sciences, 104 (11), 4730-4735. doi:10.1073/pnas.0611615104

Raghothama, K. G. (1999). PHOSPHATE ACQUISITION. Annual Review of Plant Physiology and Plant Molecular Biology, 50 (1), 665-693. doi:10.1146/annurev.arplant.50.1.665

Rubio, V., Linhares, F., Solano, R., Martin, A. C., Iglesias, J., Leyva, A., \& Paz-Ares, J. (2001). A conserved MYB transcription factor involved in phosphate starvation signaling both in vascular plants and in unicellular algae. Genes Dev, 15 (16), 2122-2133. doi:10.1101/gad.204401

Salazar-Henao, J. E., Velez-Bermudez, I. C., \& Schmidt, W. (2016). The regulation and plasticity of root hair patterning and morphogenesis.Development, 143 (11), 1848-1858. doi:10.1242/dev.132845

Saleh, A., Alvarez-Venegas, R., \& Avramova, Z. (2008). An efficient chromatin immunoprecipitation (ChIP) protocol for studying histone modifications in Arabidopsis plants. Nat Protoc, 3 (6), 1018-1025. doi:10.1038/nprot.2008.66

Schiefelbein, J. W. (2000). Constructing a Plant Cell. The Genetic Control of Root Hair Development. Plant Physiology, 124 (4), 1525-1531. doi:10.1104/pp.124.4.1525

Singh Gahoonia, T., \& Nielsen, N. E. (2004). Root traits as tools for creating phosphorus efficient crop varieties. Plant and Soil, 260 (1), 47-57. doi:10.1023/B:PLSO.0000030168.53340.bc

Song, L., Yu, H., Dong, J., Che, X., Jiao, Y., \& Liu, D. (2016). The Molecular Mechanism of EthyleneMediated Root Hair Development Induced by Phosphate Starvation. PLoS genetics, 12 (7), e1006194e1006194. doi:10.1371/journal.pgen.1006194

Svistoonoff, S., Creff, A., Reymond, M., Sigoillot-Claude, C., Ricaud, L., Blanchet, A., . . . Desnos, T. (2007). Root tip contact with low-phosphate media reprograms plant root architecture. Nat Genet, 39 (6), 792-796. doi:10.1038/ng2041

Tan, Y.-Q., Yang, Y., Zhang, A., Fei, C.-F., Gu, L.-L., Sun, S.-J., . . . Wang, Y.-F. (2020). Three CNGC Family Members, CNGC5, CNGC6, and CNGC9, Are Required for Constitutive Growth of Arabidopsis Root Hairs as Ca2+-Permeable Channels. Plant Communications, 1 (1), 100001. doi:https://doi.org/10.1016/j.xplc.2019.100001 
Thor, K. (2019). Calcium-Nutrient and Messenger. Frontiers in Plant Science, 10 , 440-440. doi:10.3389/fpls.2019.00440

Tian, W., Wang, C., Gao, Q., Li, L., \& Luan, S. (2020). Calcium spikes, waves and oscillations in plant development and biotic interactions. Nature Plants, 6 (7), 750-759. doi:10.1038/s41477-020-0667-6

Tunc-Ozdemir, M., Rato, C., Brown, E., Rogers, S., Mooneyham, A., Frietsch, S., . . . Harper, J. F. (2013). Cyclic nucleotide gated channels 7 and 8 are essential for male reproductive fertility.PloS one, 8 (2), e55277. doi:10.1371/journal.pone.0055277

Vijayakumar, P., Datta, S., \& Dolan, L. (2016). ROOT HAIR DEFECTIVE SIX-LIKE4 (RSL4) promotes root hair elongation by transcriptionally regulating the expression of genes required for cell growth. The New Phytologist, 212 (4), 944-953. doi:10.1111/nph.14095

Vissenberg, K., Claeijs, N., Balcerowicz, D., \& Schoenaers, S. (2020). Hormonal regulation of root hair growth and responses to the environment in Arabidopsis. Journal of Experimental Botany, 71 (8), 2412 2427. doi:10.1093/jxb/eraa048

Waadt, R., Schlucking, K., Schroeder, J. I., \& Kudla, J. (2014). Protein Fragment Bimolecular Fluorescence Complementation Analyses for the In vivo Study of Protein-Protein Interactions and Cellular Protein Complex Localizations. In J. J. Sanchez-Serrano \& J. Salinas (Eds.), Arabidopsis Protocols (pp. 629-658). Totowa, NJ: Humana Press.

Wang, T., Li, C., Wu, Z., Jia, Y., Wang, H., Sun, S., . . W Wang, X. (2017). Abscisic Acid Regulates Auxin Homeostasis in Rice Root Tips to Promote Root Hair Elongation. Frontiers in Plant Science, 8 (1121). doi:10.3389/fpls.2017.01121

Williamson, L. C., Ribrioux, S. P. C. P., Fitter, A. H., \& Leyser, H. M. O. (2001). Phosphate Availability Regulates Root System Architecture in Arabidopsis. Plant Physiology, 126 (2), 875-882. doi:10.1104/pp.126.2.875

Won, S.-K., Lee, Y.-J., Lee, H.-Y., Heo, Y.-K., Cho, M., \& Cho, H.-T. (2009). cis-Element- and Transcriptome-Based Screening of Root Hair-Specific Genes and Their Functional Characterization in Arabidopsis. Plant Physiology, 150 (3), 1459-1473. doi:10.1104/pp.109.140905

Yi, K., Menand, B., Bell, E., \& Dolan, L. (2010). A basic helix-loop-helix transcription factor controls cell growth and size in root hairs. Nat Genet, 42 (3), 264-267. doi:10.1038/ng.529

Ying, S., Blancaflor, E., Liao, F., \& Scheible, W. R. (2021). A Phosphorus-Limitation Induced, Functionally Conserved DUF506 Protein is a Repressor of Root Hair Elongation in Arabidopsis thaliana.bioRxiv , 2021.2007.2009.451837. doi:10.1101/2021.07.09.451837

Yu, X., Xu, G., Li, B., de Souza Vespoli, L., Liu, H., Moeder, W., . . . Shan, L. (2019). The Receptor Kinases BAK1/SERK4 Regulate $\mathrm{Ca}^{2+}$ Channel-Mediated Cellular Homeostasis for Cell Death Containment. Current Biology, 29 (22), 3778-3790.e3778. doi:https://doi.org/10.1016/j.cub.2019.09.018

Yuan, P., Jauregui, E., Du, L., Tanaka, K., \& Poovaiah, B. W. (2017). Calcium signatures and signaling events orchestrate plant-microbe interactions. Curr Opin Plant Biol, 38 , 173-183. doi:10.1016/j.pbi.2017.06.003

Zeb, Q., Wang, X., Hou, C., Zhang, X., Dong, M., Zhang, S., . . . Liu, L. (2020). The interaction of CaM7 and CNGC14 regulates root hair growth in Arabidopsis. Journal of Integrative Plant Biology, 62 (7), 887-896. doi:https://doi.org/10.1111/jipb.12890

Zhang, S., Pan, Y., Tian, W., Dong, M., Zhu, H., Luan, S., \& Li, L. (2017). Arabidopsis CNGC14 Mediates Calcium Influx Required for Tip Growth in Root Hairs. Mol Plant, 10 (7), 1004-1006. doi:10.1016/j.molp.2017.02.007 
Zhang, X., Henriques, R., Lin, S. S., Niu, Q. W., \& Chua, N. H. (2006). Agrobacterium-mediated transformation of Arabidopsis thaliana using the floral dip method. Nat Protoc, 1 (2), 641-646. doi:10.1038/nprot.2006.97

Zhang, X., Mi, Y., Mao, H., Liu, S., Chen, L., \& Qin, F. (2020). Genetic variation in ZmTIP1 contributes to root hair elongation and drought tolerance in maize. Plant Biotechnology Journal, 18 (5), 1271-1283. doi:https://doi.org/10.1111/pbi.13290

Zhu, S., Martinez Pacheco, J., Estevez, J. M., \& Yu, F. (2020). Autocrine regulation of root hair size by the RALF-FERONIA-RSL4 signaling pathway. New Phytol, 227 (1), 45-49. doi:10.1111/nph.16497

Zielinski, R. E. (1998). CALMODULIN AND CALMODULIN-BINDING PROTEINS IN PLANTS. Annu Rev Plant Physiol Plant Mol Biol, 49 , 697-725. doi:10.1146/annurev.arplant.49.1.697

\section{FIGURE LEGENDS}

Fig. 1 . qRT-PCR analysis of Arabidopsis DUF506 genes responds to phosphate starvation. Five-day-old wild-type (WT) seedlings were transferred to fresh regular $(+\mathrm{P})$ or no phosphate $(-\mathrm{P})$ half strength MS liquid medium, then the shoots and roots were harvested separately after $24 \mathrm{hr}$. At 3954550 gene was unable to detect because of its silique-specific expression pattern. Changes of gene expression level between tissues are interpreted as described previously (Bari, Datt Pant, Stitt, \& Scheible, 2006). Expression levels are given on a log scale expressed as $40-\Delta \mathrm{CT}$, where $\triangle \mathrm{CT}$ is the difference in qRT-PCR threshold cycle number between the respective gene and the reference gene $(G A P D H)$; 40 therefore equals the expression level of $G A P D H$ ; the number 40 was chosen because the PCR run stops after 40 cycles. The fold difference in expression is $2^{\Delta \Delta " T}$ when PCR efficiency is 2 (e.g. an ordinate value of 34 represents 16 -fold lower expression than a value of 38$)$.

Fig. 2 . At1g62420 responds to phosphate starvation. A, qRT PCR analysis of At1g62420 time-course expression. Five-day-old wild-type (WT) seedlings were respectively transferred to fresh regular $(+\mathrm{P})$ or no phosphate (-P) half strength MS liquid medium, then samples were harvested at indicated time point. B, GUS staining assay of the proAt1g62420::GUS transgenic line. Five-day-old transgenic seedlings were grown on $+\mathrm{P}(\mathrm{a})$ or $-\mathrm{P}$ (b and c) half strength MS solid medium for 5 day and then stained. Scale, $1 \mathrm{~mm}$ (a and b), or $50 \mu \mathrm{m}$ (c). C, Expression changes of At1g62420 in phr1 phl1 double mutant. Three-day-old seedlings were grown and treated as described in Fig. 1. D, Expression changes of At1g62420 in response to auxin (IAA). Three-day-old seedlings were transferred to $+\mathrm{P}$, or supplemented with $1 \mu \mathrm{M}$ IAA half strength MS solid medium, then shoot and root were respectively harvested after 5 day. The data represent the mean values of three replicates $\pm \mathrm{SD}$ in $\mathrm{A}, \mathrm{C}$ and $\mathrm{D}$.

Fig. 3 . Overexpression of $R X R 3$ represses Arabidopsis root hair elongation. A, Diagram of two T-DNA insertions mutants used in the study. Exons, black boxes; UTRs, white boxes; Intron, black line. B and D, Representative images showing the effect of $R X R 3$ on root hair elongation. Three-day-old seedlings were transferred to $+\mathrm{P}$ or $-\mathrm{P}$ half strength MS solid medium, then grew vertically for 2 day. While the loss-of-function mutants (rxr3-1 and rxr3-2) grow longer root hairs, constitutively overexpressing RXR3 (proCaMV35S::RXR3 , OX-5 and 12) repress root hair growth (B). The root hair growth are resumed by complementing $R X R 3$ inrxr3-1 mutant (proRXR3::RXR3-GFP, Com\#1 and \#2, D). Scale, $1 \mathrm{~mm}$. C and E, Violin plots of root hair lengths in WT, overexpressors, rxr3 mutants, and complemented lines, under $\mathrm{P}$ sufficient or deficient condition. The plot illustrates kernel probability density in which the width represents distribution of data points. Dash lines indicate 25 th and 75 th percentiles and horizontal black line is the median value. Between 885 and 1026 root hairs from 10 seedlings were measured for each genotype and numbers at bottom indicate median values (in mm) for each genotype. F, Immunoblot analysis of RXR3-GFP fusion protein in complementation lines (\#1 and \#2) under different P-status. Coomassie-stained RuBisCO protein is shown as loading control. G, Root hair apical (a) and root epidermal (b, transection view) localization of RXR3-GFP fusion protein in Arabidopsis complemented line (Comp\#1) under P-stress. Scale, $100 \mu \mathrm{m}$. H and I, Box plots of shoot phosphate content (H) or fresh biomass (I) of 10-day-old wild type (blue) and rxr3-1 mutant (orange) seedlings that grown on $+\mathrm{P}$ or $-\mathrm{P}$ half strength $\mathrm{MS}$ solid medium $(n=3)$. Horizontal line is the 
median and whiskers display minimum and maximum values. C, E, $\mathrm{H}$ and I, ${ }^{* * * *} P<0.0001,{ }^{* * *} P<0.001$, ${ }^{* *} P<0.01$ or $* P<0.05$ indicates statistical significance as determined by one-way ANOVA analysis. Ns, no significance.

Fig. 4 . Root hair phenotype of $r x r 1$ and $r x r 3-1$ single mutant, and $r x r 1$ rxr3 double mutant. A, Representative root hair images of 5-day-old seedlings. Scale, $1 \mathrm{~mm}$. B, Violin plots of root hair lengths in WT, $\operatorname{rxr} 1$ and rxr3-1 single mutants, and $\operatorname{rxr} 1$ rxr3 double mutant, under $\mathrm{P}$ sufficient or deficient condition. The plot illustrates kernel probability density in which the width represents distribution of data points. Dash lines indicate 25th and 75th percentiles and horizontal black line is the median value. Between 906 and 1037 root hairs from 10 seedlings were measured for each genotype and numbers at bottom indicate median values (in $\mathrm{mm}$ ) for each genotype. ${ }^{* * * *} P<0.0001,{ }^{* * *} P<0.001$, or ${ }^{* *} P<0.01$ indicates statistical significance as determined by one-way ANOVA analysis. Ns, no significance.

Fig.5 . RSL4 binds to the $R X R 3$ promoter. A, Diagram of the $R X R 3$ promoter region showing the relative positions of the RHEs. The RHEs are marked by gray rectangles, and relative positions and sizes of the different PCR-amplified fragments are indicated by black lines under the RHE(s). B, qRT PCR analysis of $R X R 3$ expression inrsl4 mutant. Five-day-old Arabidopsis seedlings were transferred to fresh $+\mathrm{P}$ or -P half strength MS liquid medium, then the roots were harvested after $24 \mathrm{hr}$. The data represent the mean values of three replicates \pm SD. C, ChIP analysis to detect the association between RSL4 and the RHEs within the RXR3 promoter in the RSL4-GFP transgenic line. The ChIP signals with (anti-GFP) and without (No Ab) addition of anti-GFP are indicated. D, Nucleus-localized GFP signal (Z-stack) in roots of rsl4 complementation line (Comp\#1) harboringproRSL4::RSL4-GFP . Scale, $30 \mu \mathrm{m}$.

Fig.6 . RXR3 responds to AtRALF1. A, Representative images showing the effect of RALF1 on root hair elongation. Three-day-old seedlings were transferred to $+\mathrm{P}$, or supplemented with $1 \mu \mathrm{M}$ RALF1 half strength MS solid medium, then grew vertically for 2 day. Both WT and the rxr3-1 mutant grow longer root hairs, under RALF1 treatment. Scale, 1mm. B, qRT PCR analysis of RXR3 expression in rsl4 mutant, under RALF1 treatment. Three-day-old seedlings (WT and rsl4) were transferred and treated as described in (A), and then the roots were harvested after 5 day. The data represent the mean values of three replicates \pm SD. C, GUS staining assay of theproRXR3::GUS transgenic line under $+\mathrm{P}$ or RALF1 treatment (as described in A). Scale, $100 \mu \mathrm{m}$. D, Root hair apical (a) and root epidermal (b, transection view) localization of RXR3-GFP fusion protein in Arabidopsis complemented line (Comp \#1) under RALF1 treatment. Scale, $100 \mu \mathrm{m}$.

Fig.7 . RXR3 interacts with CaMs. A, Diagram of full-length (FL) RXR3 protein tagged with maltose binding protein (MBP, black boxes), and its truncated mutant ( $\Delta 1-22)$ which lacks of Calmodulin-binding domain (red box). Green boxes, the three conserved motifs present in plant DUF506 family (Ying et al., 2021). B, Immunoblot analysis of recombinant MBP-RXR3 ${ }^{\mathrm{FL}}$ and MBP-RXR3 ${ }^{\Delta 1-22}$ protein binding with CaM-agarose in presence or absence of $\mathrm{Ca}^{2+}$. Recombinant protein $(2 \mu \mathrm{g}$ each) were incubated with CaMagarose in the presence of $0.5 \mathrm{mM} \mathrm{Ca}^{2+}$ or $2 \mathrm{mM}$ EGTA. Proteins bound to the CaM-agarose were separated by SDS-PAGE gel, and detected by immunoblotting with anti-MBP tag antibody. C, BiFC analysis to detect the interaction between RXR3 and CaMs in N. benthamiana. Full-length of RXR3 or RXR1 (as control), and various CaM genes were respectively constructed into pSITE-cEYFP or pSITE-nEYFP vectors. Then, constructs were co-transformed into $N$. benthamianaleaves via syringe-infiltration. Scale, $20 \mu \mathrm{m}$.

Fig.8 . RXR3 affects root hair apical $\left[\mathrm{Ca}^{2+}\right]_{\text {cyt }}$ oscillation. A, BiFC analysis to confirm the interaction between CNGC14 and CaM7 in N. benthamiana. B, BiFC analysis to investigate the interaction between CNGC14 and CaM7 is independent of RXR3. Arrow, nucleus. Scale, $20 \mu \mathrm{m}$ (A) or $40 \mu \mathrm{m}$ (B). C, Representative normalized oscillograms of wild type andrxr3-1 mutant. The present data has been processed by baseline simulation analysis. D, Box plot of $\left[\mathrm{Ca}^{2+}\right]$ oscillations frequencies in root hairs of rxr3 mutants and wild type over $600 \mathrm{sec}$. Box limits indicate $25 \mathrm{th}$ and 75 th percentiles, horizontal line is the median, and whiskers display minimum and maximum values. Each semitransparent dot represents individual measurements from 7 to 11 root hairs per group from 4 to 6 plants. ${ }^{* *} P<0.01$ indicates statistical significance as determined by Student's $t$ test. Ns, no significance. 
Fig.9 . Proposed working model that illustrating the different pathway between RXR1 and RXR3 in repressing root hair elongation. Under P-stress, $R X R 3$ is transcriptionally activated by auxin through RSL4 $\mathrm{TF}$, whereas $R X R 1$ is participating in PHR1/PHL1-dependent pathway. RXR1 interacts with RabD2c GTPase to inhibit RH elongation. Here, RXR3 binds to $\mathrm{Ca}^{2+}$ activated CaMs to regulate $\left[\mathrm{Ca}^{2+}\right]_{\text {cyt }}$ oscillation and eventually $\mathrm{RH}$ growth. Blunt end, negative action; arrow, positive action. Solid line, experimentally supported; broken line, hypothetical.

\section{FIGURE}

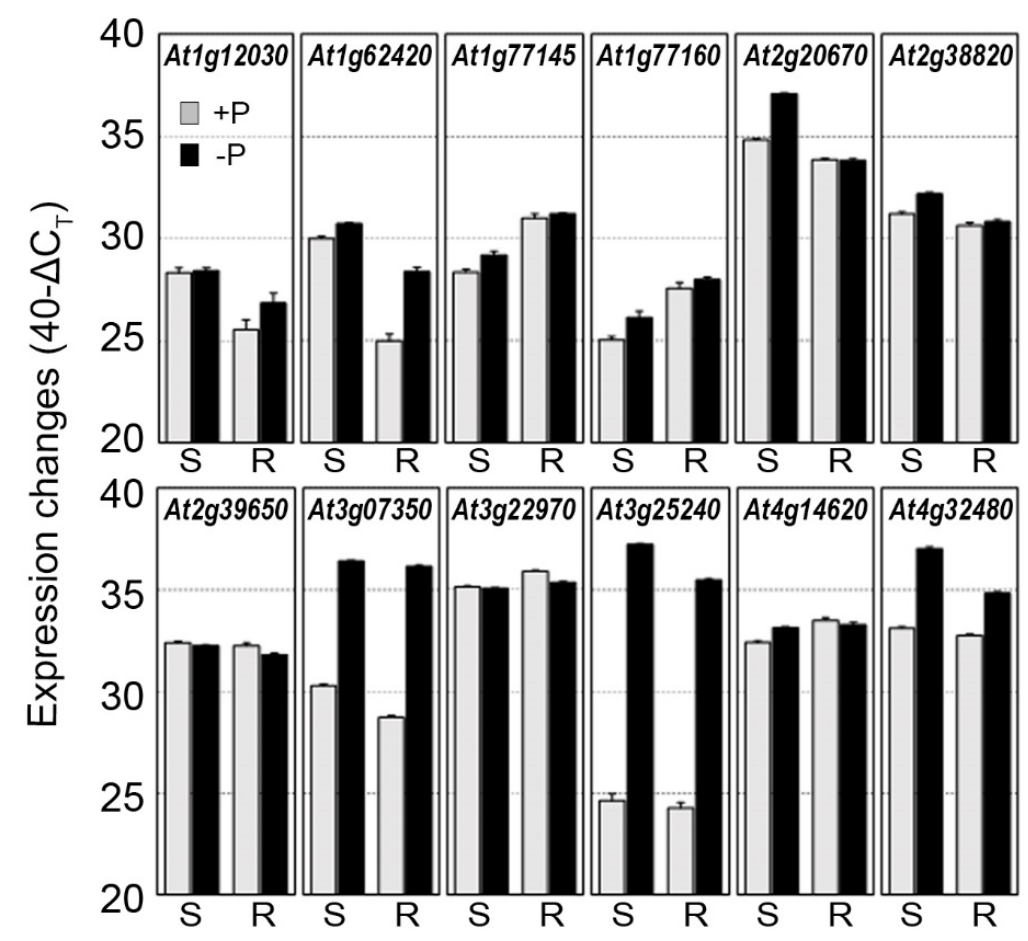

Fig. 1 . qRT-PCR analysis of Arabidopsis DUF506 genes responds to phosphate starvation. Five-day-old wild-type (WT) seedlings were transferred to fresh regular $(+\mathrm{P})$ or no phosphate $(-\mathrm{P})$ half strength MS liquid medium, then the shoots and roots were harvested separately after $24 \mathrm{hr}$. At3g54550 gene was unable to detect because of its silique-specific expression pattern. Changes of gene expression level between tissues are interpreted as described previously (Bari et al., 2006). Expression levels are given on a log scale expressed as $40-\Delta \mathrm{CT}$, where $\triangle \mathrm{CT}$ is the difference in qRT-PCR threshold cycle number between the respective gene and the reference gene $(G A P D H)$; 40 therefore equals the expression level of $G A P D H$; the number 40 was chosen because the PCR run stops after 40 cycles. The fold difference in expression is $2^{\Delta \Delta} \mathrm{T}$ when PCR efficiency is 2 (e.g. an ordinate value of 34 represents 16 -fold lower expression than a value of 38). 
A

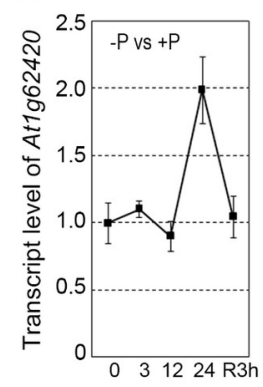

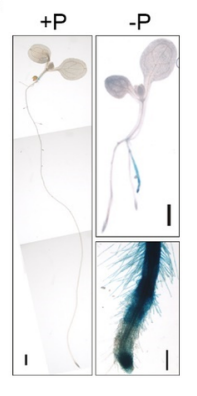

C

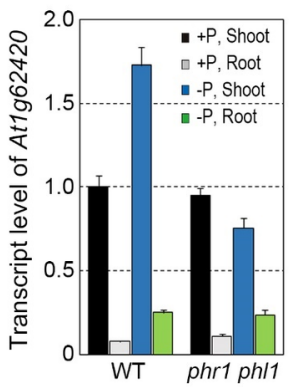

D

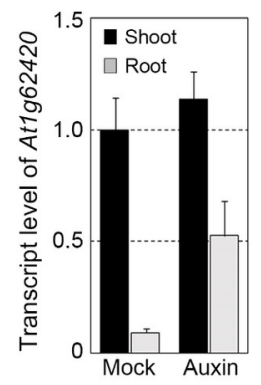

Fig. 2 . At1g62420 responds to phosphate starvation. A, qRT PCR analysis of At1g62420 time-course expression. Five-day-old wild-type (WT) seedlings were respectively transferred to fresh regular $(+\mathrm{P})$ or no phosphate (-P) half strength MS liquid medium, then samples were harvested at indicated time point. B, GUS staining assay of the proAt1g62420::GUS transgenic line. Five-day-old transgenic seedlings were grown on $+\mathrm{P}$ (a) or $-\mathrm{P}$ (b and c) half strength MS solid medium for 5 day and then stained. Scale, $1 \mathrm{~mm}$ (a and b), or $50 \mu \mathrm{m}$ (c). C, Expression changes of At1g62420 in phr1 phl1 double mutant. Three-day-old seedlings were grown and treated as described in Fig. 1. D, Expression changes of At1g62420 in response to auxin (IAA). Three-day-old seedlings were transferred to $+\mathrm{P}$, or supplemented with $1 \mu \mathrm{M}$ IAA half strength MS solid medium, then shoot and root were respectively harvested after 5 day. The data represent the mean values of three replicates $\pm \mathrm{SD}$ in $\mathrm{A}, \mathrm{C}$ and $\mathrm{D}$. 

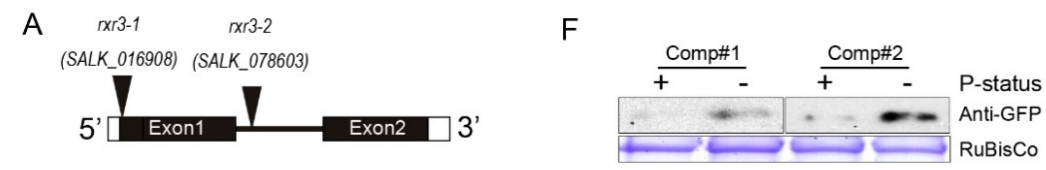

B
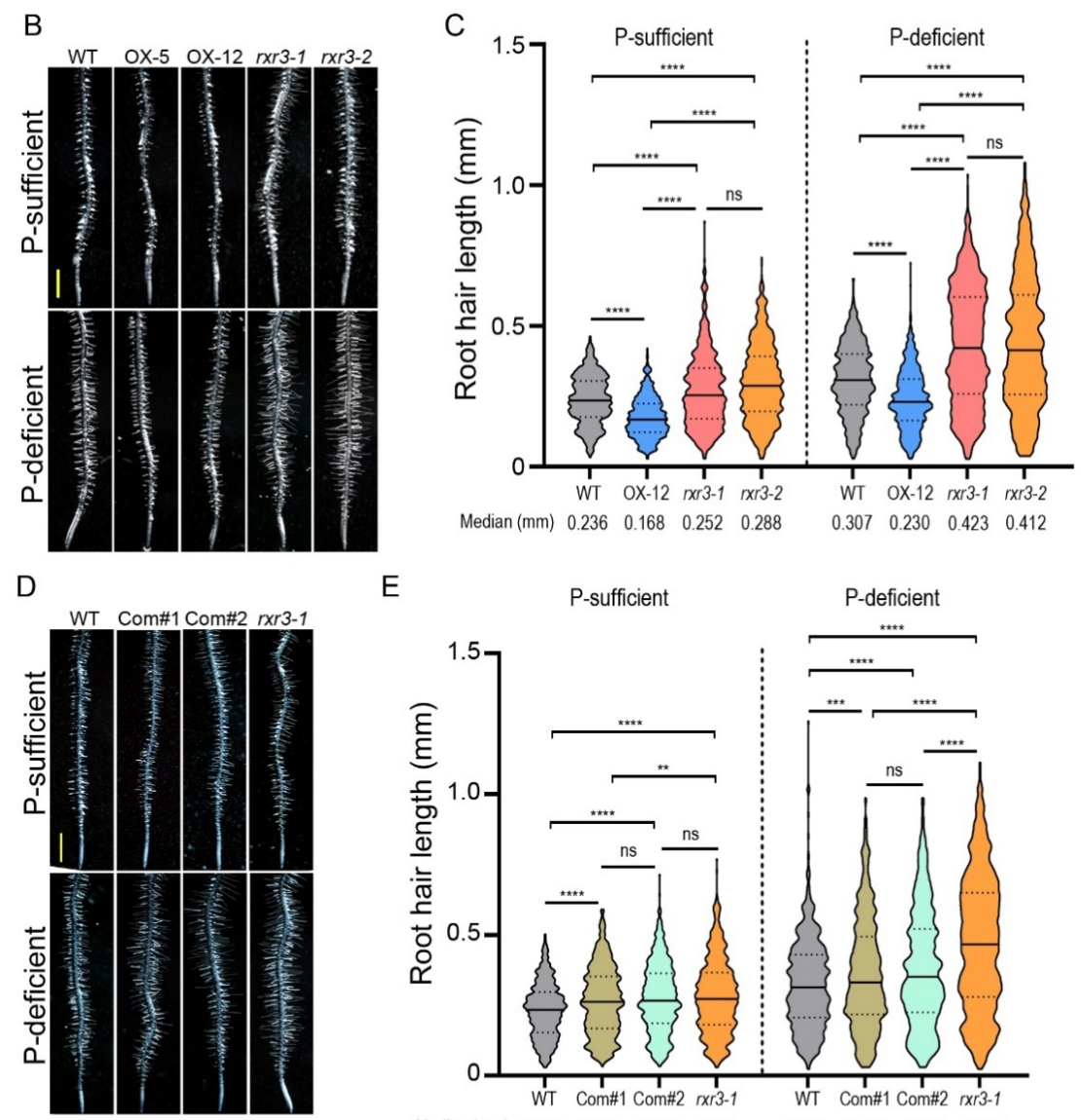

E

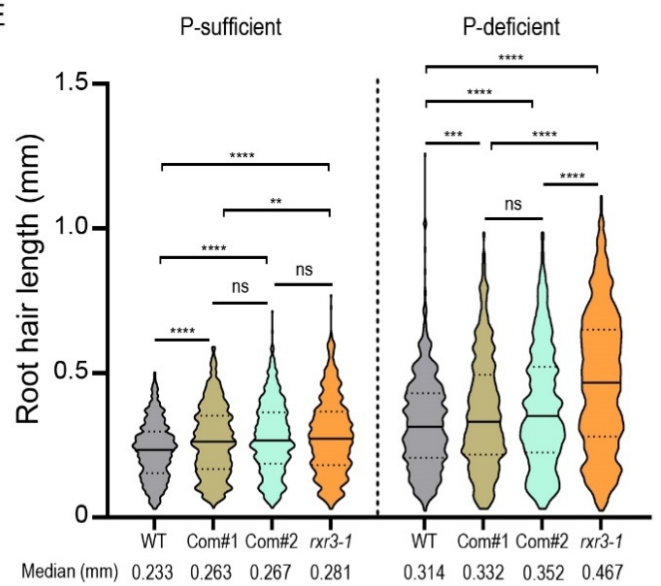

G

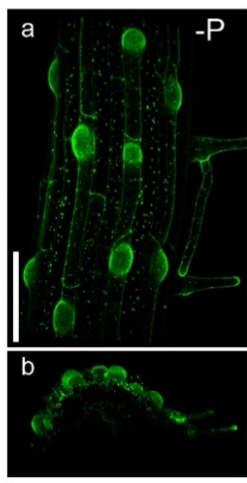

$\mathrm{H}$
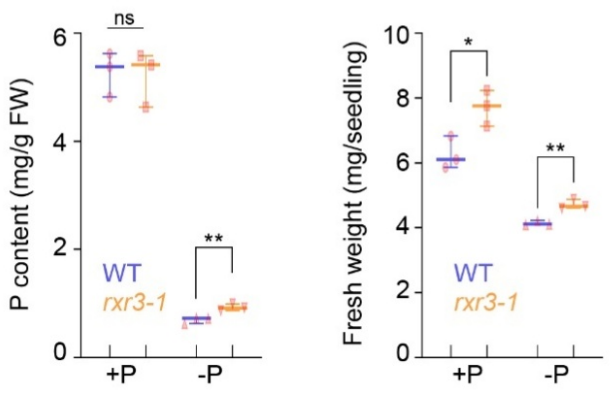

Fig. 3 . Overexpression of $R X R 3$ represses Arabidopsis root hair elongation. A, Diagram of two TDNA insertions mutants used in the study. Exons, black boxes; UTRs, white boxes; Intron, black line. $\mathrm{B}$ and D, Representative images showing the effect of $R X R 3$ on root hair elongation. Three-day-old seedlings were transferred to $+\mathrm{P}$ or $-\mathrm{P}$ half strength $\mathrm{MS}$ solid medium, then grew vertically for 2 day. 
While the loss-of-function mutants (rxr3-1 and rxr3-2 ) grow longer root hairs, constitutively overexpressing RXR3 (proCaMV35S::RXR3, OX-5 and 12) repress root hair growth (B). The root hair growth are resumed by complementing $R X R 3$ inrxr3-1 mutant (proRXR3::RXR3-GFP , Com\#1 and \#2, D). Scale, 1 $\mathrm{mm}$. C and E, Violin plots of root hair lengths in WT, overexpressors, rxr3 mutants, and complemented lines, under $\mathrm{P}$ sufficient or deficient condition. The plot illustrates kernel probability density in which the width represents distribution of data points. Dash lines indicate 25th and 75 th percentiles and horizontal black line is the median value. Between 885 and 1026 root hairs from 10 seedlings were measured for each genotype and numbers at bottom indicate median values (in $\mathrm{mm}$ ) for each genotype. F, Immunoblot analysis of RXR3-GFP fusion protein in complementation lines (\#1 and \#2) under different P-status. Coomassiestained RuBisCO protein is shown as loading control. G, Root hair apical (a) and root epidermal (b, transection view) localization of RXR3-GFP fusion protein in Arabidopsis complemented line (Comp\#1) under P-stress. Scale, $100 \mu \mathrm{m}$. H and I, Box plots of shoot phosphate content (H) or fresh biomass (I) of 10-day-old wild type (blue) and rxr3-1 mutant (orange) seedlings that grown on $+\mathrm{P}$ or $-\mathrm{P}$ half strength MS solid medium $(n=3)$. Horizontal line is the median and whiskers display minimum and maximum values. $\mathrm{C}, \mathrm{E}, \mathrm{H}$ and I, ${ }^{* * * *} P<0.0001,{ }^{* * *} P<0.001,{ }^{* *} P<0.01$ or ${ }^{*} P<0.05$ indicates statistical significance as determined by one-way ANOVA analysis. Ns, no significance.
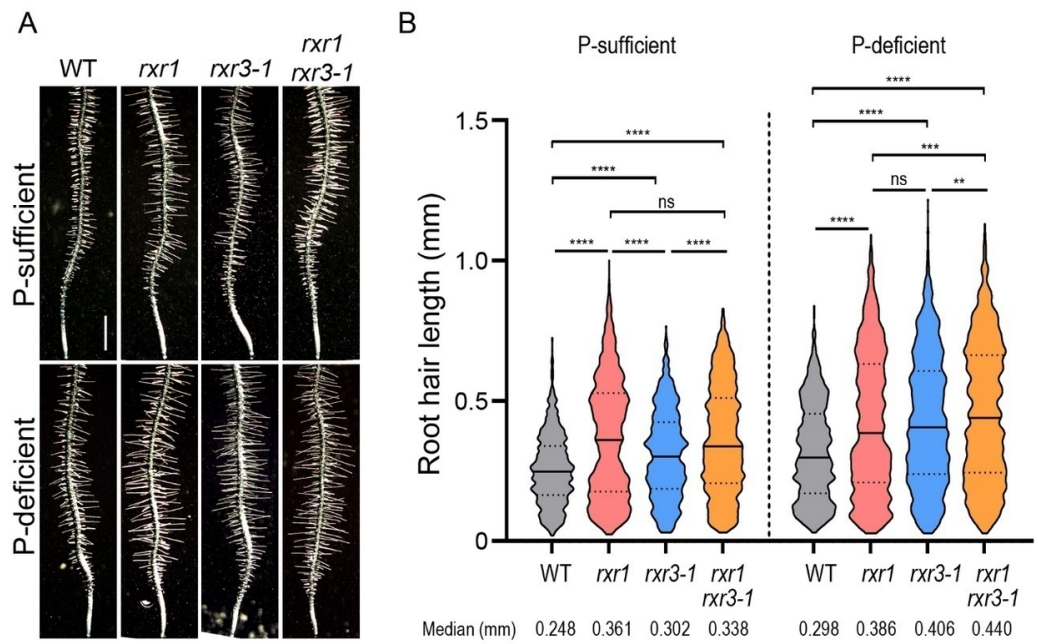

Fig. 4. Root hair phenotype of $\operatorname{rxr} 1$ and $\operatorname{rxr} 3-1$ single mutant, and $\operatorname{rxr} 1$ rxr3 double mutant. A, Representative root hair images of 5-day-old seedlings. Scale, $1 \mathrm{~mm}$. B, Violin plots of root hair lengths in WT, rxr1 and rxr3-1 single mutants, and rxr1 rxr3double mutant, under P sufficient or deficient condition. The plot illustrates kernel probability density in which the width represents distribution of data points. Dash lines indicate 25th and 75th percentiles and horizontal black line is the median value. Between 906 and 1037 root hairs from 10 seedlings were measured for each genotype and numbers at bottom indicate median values (in $\mathrm{mm}$ ) for each genotype. ${ }^{* * * *} P<0.0001,{ }^{* * *} P<0.001$, or ${ }^{* *} P<0.01$ indicates statistical significance as determined by one-way ANOVA analysis. Ns, no significance. 

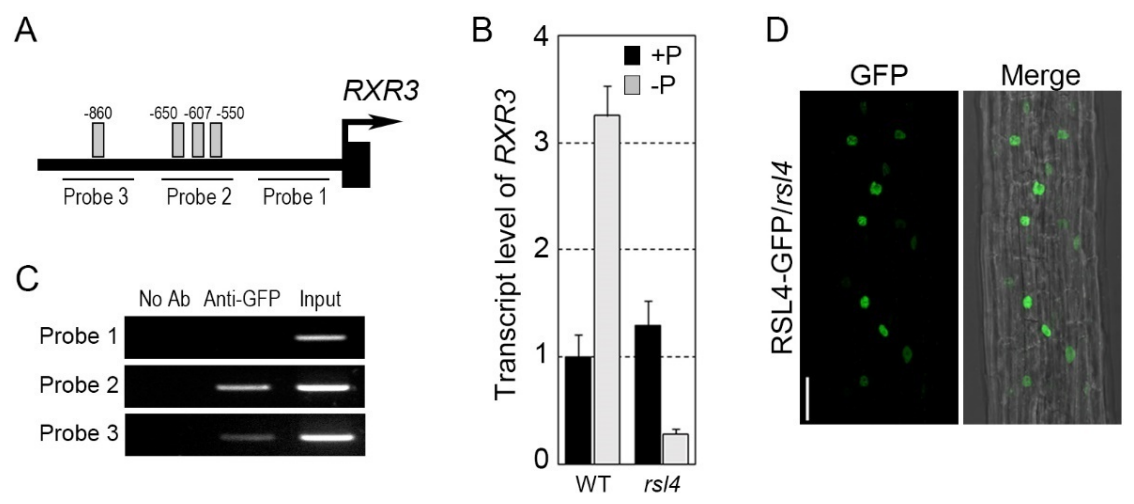

Fig. 5 . RSL4 binds to the RXR3 promoter. A, Diagram of the $R X R 3$ promoter region showing the relative positions of the RHEs. The RHEs are marked by gray rectangles, and relative positions and sizes of the different PCR-amplified fragments are indicated by black lines under the RHE(s). B, qRT PCR analysis of $R X R 3$ expression in rsl4 mutant. Five-day-old Arabidopsis seedlings were transferred to fresh $+\mathrm{P}$ or $-\mathrm{P}$ half strength MS liquid medium, then the roots were harvested after $24 \mathrm{hr}$. The data represent the mean values of three replicates $\pm \mathrm{SD}$. C, ChIP analysis to detect the association between RSL4 and the RHEs within the RXR3 promoter in the RSL4-GFP transgenic line. The ChIP signals with (anti-GFP) and without (No Ab) addition of anti-GFP are indicated. D, Nucleus-localized GFP signal (Z-stack) in roots of rsl4 complementation line (Comp\#1) harboringproRSL4::RSL4-GFP . Scale, $30 \mu \mathrm{m}$.
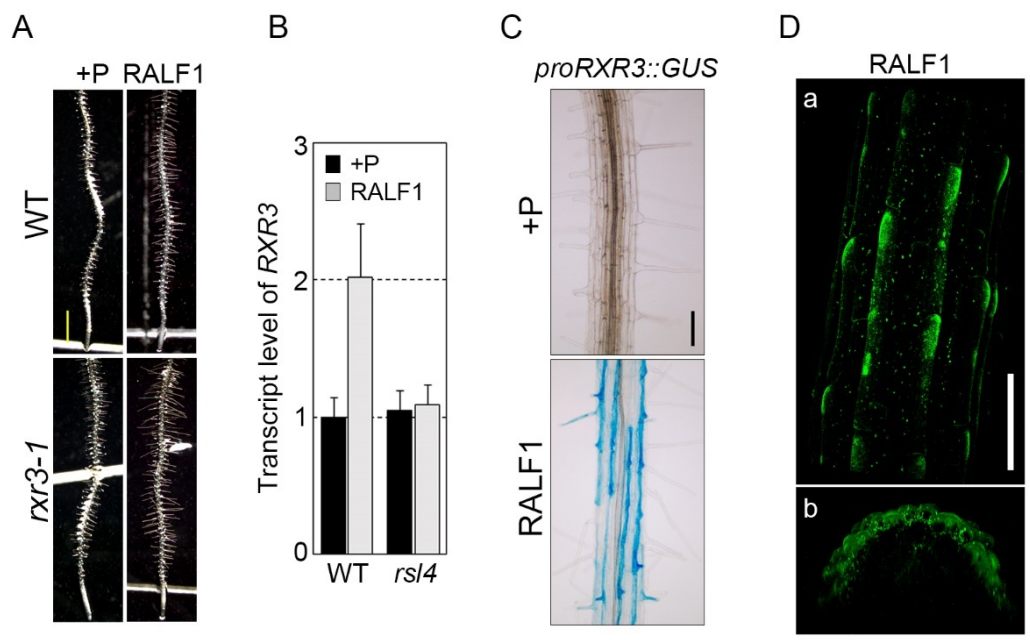

Fig. 6 . RXR3 responds to AtRALF1. A, Representative root images showing the effect of RALF1 on

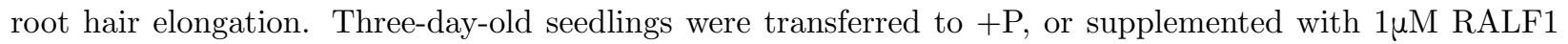
half strength MS solid medium, then grew vertically for 2 day. Both WT and the rxr3-1 mutant grow longer root hairs, under RALF1 treatment. Scale, $1 \mathrm{~mm}$. B, qRT PCR analysis of RXR3 expression in rsl/4 mutant, under RALF1 treatment. Three-day-old seedlings (WT and $r s l_{4}$ ) were transferred and treated as described in (A), and then the roots were harvested after 5 day. The data represent the mean values of three replicates $\pm \mathrm{SD}$. C, GUS staining assay of theproRXR3::GUS transgenic line under $+\mathrm{P}$ or RALF1 treatment (as described in A). Scale, $100 \mu \mathrm{m}$. D, Root hair apical (a) and root epidermal (b, transection view) localization of RXR3-GFP fusion protein in Arabidopsis complemented line (Comp \#1) under RALF1 treatment. Scale, $100 \mu \mathrm{m}$. 
A

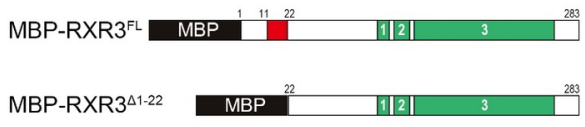

B

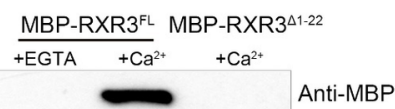

C

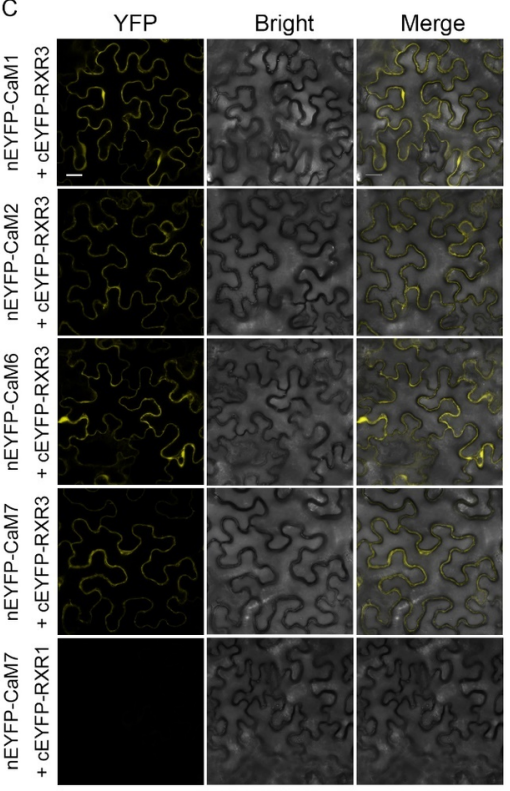

Fig. 7 . RXR3 interacts with CaMs. A, Diagram of full-length (FL) RXR3 protein tagged with maltose binding protein (MBP, black boxes), and its truncated mutant ( $\Delta 1-22)$ which lacks of Calmodulin-binding domain (red box). Green boxes, the three conserved motifs present in plant DUF506 family (Ying et al., 2021). B, Immunoblot analysis of recombinant MBP-RXR3 $3^{\mathrm{FL}}$ and MBP-RXR3 ${ }^{\Delta 1-22}$ protein binding with CaM-agarose in presence or absence of $\mathrm{Ca}^{2+}$. Recombinant protein $(2 \mu \mathrm{g}$ each) were incubated with CaMagarose in the presence of $0.5 \mathrm{mM} \mathrm{Ca}^{2+}$ or $2 \mathrm{mM}$ EGTA. Proteins bound to the CaM-agarose were separated by SDS-PAGE gel, and detected by immunoblotting with anti-MBP tag antibody. C, BiFC analysis to detect the interaction between RXR3 and CaMs in N. benthamiana. Full-length of RXR3 or RXR1 (as control), and various CaM genes were respectively constructed into pSITE-cEYFP or pSITE-nEYFP vectors. Then, constructs were co-transformed into $N$. benthamiana leaves viasyringe-infiltration. Scale, $20 \mu \mathrm{m}$.
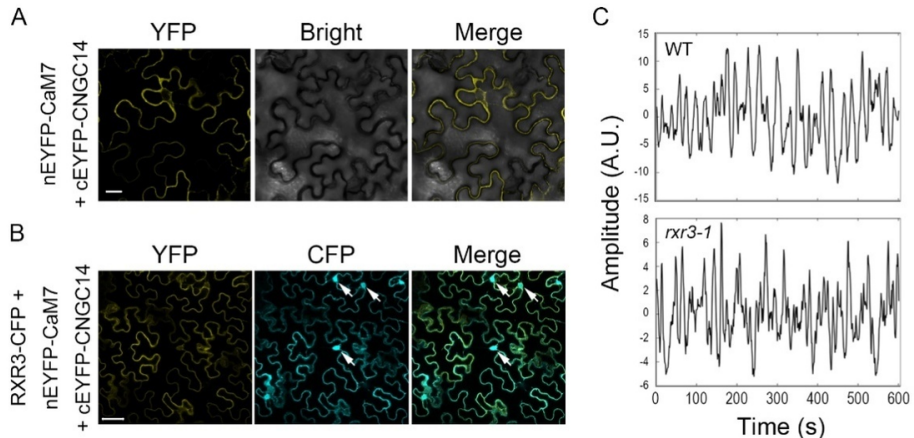

D

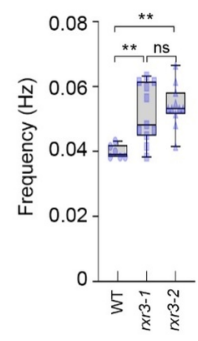

Fig. 8. RXR3 affects root hair apical $\left[\mathrm{Ca}^{2+}\right]_{\text {cyt }}$ oscillation. A, BiFC analysis to confirm the interaction between CNGC14 and CaM7 in N. benthamiana. B, BiFC analysis to investigate the interaction between CNGC14 and CaM7 is independent of RXR3. Arrow, nucleus. Scale, $20 \mu \mathrm{m}$ (A) or $40 \mu \mathrm{m}$ (B). C, Representative normalized oscillograms of wild type and $r x r 3-1$ mutant. The present data has been processed by baseline simulation analysis. D, Box plot of $\left[\mathrm{Ca}^{2+}\right]_{\mathrm{cyt}}$ oscillations frequencies in root hairs of rxr3 mutants 
and wild type over $600 \mathrm{sec}$. Box limits indicate 25 th and 75 th percentiles, horizontal line is the median, and whiskers display minimum and maximum values. Each semitransparent dot represents individual measurements from 7 to 11 root hairs per group from 4 to 6 plants. ${ }^{*} P<0.01$ indicates statistical significance as determined by one-way ANOVA analysis. Ns, no significance.

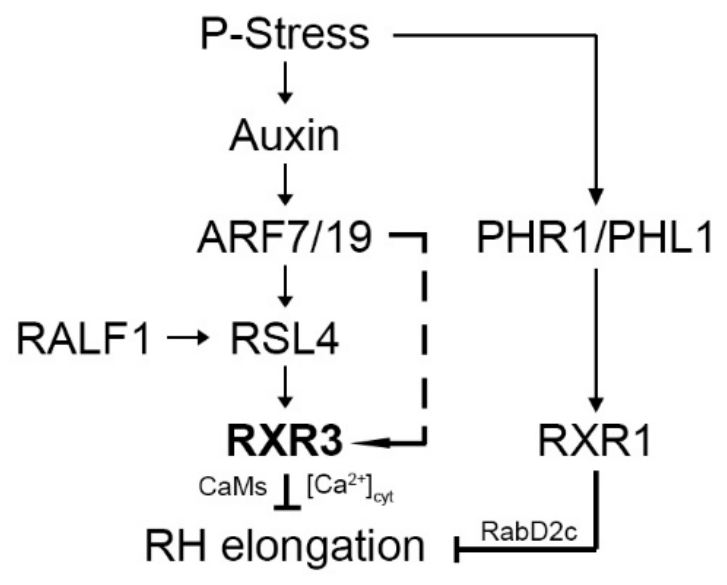

Fig. 9. Proposed working model that illustrating the different pathway between RXR1 and RXR3 in repressing root hair elongation. Under P-stress, $R X R 3$ is transcriptionally activated by auxin through RSL4 TF, whereas $R X R 1$ is participating in PHR1/PHL1-dependent pathway. RXR1 interacts with RabD2c GTPase to inhibit RH elongation. Here, RXR3 binds to $\mathrm{Ca}^{2+}$ activated CaMs to regulate $\left[\mathrm{Ca}^{2+}\right]_{\text {cyt }}$ oscillation and eventually RH growth. Blunt end, negative action; arrow, positive action. Solid line, experimentally supported; broken line, hypothetical.

\section{Hosted file}

FIGURE.docx available at https://authorea.com/users/435313/articles/538422-a-novelcalmodulin-interacting-domain-of-unknown-function-506-protein-represses-root-hairelongation-in-arabidopsis 